\title{
Educación infantil, ideología y clase social. Una lectura al sur de Europa a través de los datos del CIS para España
}

\author{
Early Childhood Education, Ideology and Social Class. A description of \\ southern Eu-rope through the CIS data for Spain \\ Daniel Gabaldón Estevan y Kadri Täht'
}

\section{Resumen}

A diferencia del modelo nórdico, en el que junto a los permisos parentales de larga duración predomina la oferta pública y privada subsidiada de centros de educación infantil, la realidad del sur de Europa es menos favorable, desde el punto de vista institucional, a la conciliación de la vida familiar y laboral. No obstante, la escolarización en la primera etapa de la educación infantil (0-2 años) ha mostrado un incremento sostenido en los últimos años también en los países del sur de Europa, aunque, en este contexto, poco se sabe acerca de las razones que justifican o no la escolarización de los más pequeños. El objetivo de la presente investigación es estudiar si existen diferencias entre la población española en general, y las familias en particular, en función de su ideología y clase social, respecto del papel que otorgan a la educación infantil en el desarrollo de niñas y niños. Para ello esta investigación se centra exclusivamente en la explotación del estudio del Centro de Investigaciones Sociológicas (CIS) n. ${ }^{\circ}$ 2788-Barómetro de febrero de 2009. Los resultados muestran que las diferencias entre ideología y clase social nos ayudan a entender la diferente apuesta que hacen los individuos encuestados no solo acerca de la matriculación o no de sus menores en escuelas infantiles de primer ciclo, sino también respecto de cuestiones relativas a las prioridades de la administración en esta materia, o a la provisión pública o privada de estos servicios.

\section{Palabras clave}

Educación infantil, ideología, clase social, transición educativa.

\section{Abstract}

Unlike the Nordic model, in which, along with long-term parental leave, the subsidized public and private supply of pre-school education centers predominates, the reality of southern Europe is less favorable, from the institutional point of view, to the reconcilia-tion of family and work life. However, schooling in the first stage of early childhood education (0-2 years) has shown a sustained increase in recent years also in the coun-tries of southern Europe, although, in this context, little is known about the reasons that justify or not the schooling of children. The objective of the present investigation is to study if there are differences between the Spanish population in general, and families in particular, according to their ideology and social class, regarding the role they give to early childhood education in the development of girls and boys. For this, this research focuses exclusively on the exploitation of the study of the Center for Sociological Re-search (CIS) n. ${ }^{\circ}$ 2788-Barometer of February 2009. The results show that the differences between ideology and social class help us understand the different bet that individuals make respondents not only about the enrollment or not of their children in early child-hood schools, but also regarding issues related to the priorities of the administration in this matter, or to the public or private provision of these services.

\section{Keywords}

Early childhood education, ideology, social class, educational transition.

\section{Cómo citar/Citation}

Gabaldón Estevan, Daniel y Täht, Kadri (2019). Educación infantil, ideología y clase social. Una lectura al sur de Europa a través de los datos del CIS para España. Revista de Sociologia de la Educación-RASE, 12 (3), 367-394. http://dx.doi.org/10.7203/RASE.12.3.15760. 


\section{Introducción}

El creciente interés acerca de la visión que la sociedad, europea en general y española en particular, tienen acerca de la escolarización temprana de sus menores radica en que es un fenómeno relativamente reciente en el sur de Europa, muy dependiente de las redes familiares y de parentesco para suplir las carencias de un estado del bienestar menos desarrollado que el de nuestros vecinos del norte (Ancheta, 2011). Máxime porque la familia y la escuela son los contextos de desarrollo y educación por excelencia y la importancia que estos ambientes educativos tienen en el desarrollo de la infancia es determinante en su desarrollo (Bowlby, 2005; Vila, 1995; Cebolla-Boado et al., 2014).

Como pone de manifiesto González (2005), los servicios que surgieron como respuesta a las necesidades derivadas de la expansión del empleo femenino remunerado y los cambios en las estructuras familiares tradicionales fueron ganando aceptación y se generó un amplio consenso en cuanto a que el acceso a los servicios de calidad promueve la igualdad de oportunidades. Así, de igual manera que se prolonga la educación obligatoria de los 12 a los 14 con la Ley General de Educación (LGE) de 1970, y de los 14 a los 16 con la Ley Orgánica General del Sistema Educativo (LOGSE) de 1990, un efecto similar, sin comportar la obligatoriedad, se produce en la educación infantil. Ya el Libro Blanco para la Reforma del Sistema Educativo de 1989 lanza la propuesta para una ampliación de la educación infantil a todo el tramo 0-6 frente a los 2-6 que en ese momento se contemplaban (Grana y Martín-Zúñiga, 2010). Si bien la LOGSE establece la Educación Infantil como una etapa (0-6) de dos ciclos (0-2 y 3-6) la ley posterior, la Ley de Orgánica de Calidad de la Educación (LOCE) de 2002, cambiara la denominación del primer ciclo de nuevo a prescolar, acotando su función a la de guardia y custodia para facilitar la conciliación de los adultos. Posteriormente la Ley Orgánica de Educación (LOE) de 2006, volverá a situar el 0-2 en el marco de la educación infantil cuya intencionalidad de etapa es netamente educativa, que no necesariamente escolar, dejando atrás la a función social de protección y cuidado que le otorgaba la LOCE. La última reforma educativa, la Ley Orgánica para la Mejora de la Calidad Educativa (LOMCE) de 2013 no legisló en materia de educación infantil, quedando por lo tanto vigente a este respecto la LOE.

Otro de los aspectos relevantes a la hora de entender la desigual evolución de la educación infantil es la cuestión de su financiamiento. Si bien, como ya se ha apuntado, no se trata de educación obligatoria, para el segundo ciclo de infantil sí que se garantiza su gratuidad. No así el primer ciclo que queda a expensas de la respuesta que las diferentes administraciones con competencias en educación infantil en España, le den, y esta ha sido muy dispar incluso a nivel municipal. A modo de ejemplo destaca la poca oferta de plazas públicas en ciudades como Alicante o Valencia (Gabaldón, 2016; Jiménez et al., 2016) incluso muy por debajo de la comunidad autónoma a la que pertenecen (Colom y Gabaldón, 2016).

No obstante, la concepción que la sociedad en general, y las familias en particular, tienen sobre lo que es mejor para sus hijas e hijos no responde a un único patrón, sino que varían tanto entre familias en un momento dado (Gabaldón, 2016) como en una sociedad determinada a lo largo del tiempo (Rodríguez, 2007; Putnam, 2015). La literatura existente atribuye parte de esta falta de homogeneidad a patrones diferenciados en los que se observan estrategias de enclasamiento y diferenciación de aquellos segmentos de la población con más recursos (Feito, 1994; Fernández-Esquinas, 2004; Mancebón, 2007; Fernández-Enguita, 2008; Colom Ortiz y Gabaldón-Estevan, 2016). Por otra parte, estas preferencias pueden responder también a concepciones diferentes del papel de la educación en la sociedad según el modelo de parentalidad o la ideología de adscripción (Fernández-Palomares, 2003; Obiol, 2011). 
No obstante, se desconocen estudios previos que vinculen la relación entre ideología y clase social y las diferentes visiones respecto de la educación infantil. Analizar las diferencias existentes respecto del papel que la ciudadanía y las familias otorgan a la educación infantil así como a qué papel juega la ideología y la clase social para explicar esas diferencias es relevante en el ámbito de las políticas sociales y educativas ya que permite conocer las diferencias existentes entre los planteamientos sociales y las elecciones parentales a la hora de iniciar la transición ${ }^{2}$, o incorporación, de hijas e hijos al sistema educativo.

\section{Metodología}

En esta investigación se realiza una explotación de los datos del estudio del CIS n. ${ }^{\circ}$ 2788-Barómetro de febrero de 2009. En particular se estudia la capacidad de las variables ideología (v27) y clase social (v44) para explicar tendencias de respuesta a las preguntas sobre opiniones respecto la importancia de la etapa 0-2 años y la atención que recibe de la sociedad en general (v12) y de las Administraciones Públicas en particular (v11, v13, v14, v15), de la conveniencia de que niñas y niños cursen los diferentes ciclos de la educación infantil (v8, v10, v16, v16a, v17, v17a, v18, v18a, v19, v20, v21), de quién es mejor proveedor de este servicio (v22, ), y de qué problemáticas identifican (v23, v24). Cómo variables de control contamos con si las encuestadas y encuestados tienen o no descendencia y las características de esta (v25, v25a y v25b) así como el tamaño del hogar (v38). Además se incluyen como controles otras más habituales como el sexo (v29), la edad (v30), nacionalidad (v41), el nivel de estudios (v31a), o el tipo de ocupación (v35), la situación actual en la que se encuentra (v34, v43) o la actividad y tipo de empresa para la que trabaja (v36a, v37). Como complemento del estudio, tomando solo aquellas/os encuestados con descendencia, se analizará la relación entre ideología (v27) y clase social (v44) con las prácticas de crianza declaradas (v26) y su justificación (v26a y v26b). La siguiente figura representa esquemáticamente las relaciones estudiadas:

Figura 1. Relaciones a estudiar en el presente trabajo

\begin{tabular}{|c|c|}
\hline $\begin{array}{l}\text { Variables de control: } \\
\text { - Descendencia y características (v25, v25a y v25b) } \\
\text { - Tamaño del hogar (v38) } \\
\text { - Sexo (v29) } \\
\text { - Edad (v30) } \\
\text { - Nacionalidad (v41) } \\
\text { - Nivel de estudios (v3la) } \\
\text { - Tipo de ocupación (v35) } \\
\text { - Situación actual entrevistado (v34 y v43) } \\
\text { - Actividad y tipo de empresa para la que trabaja } \\
\text { (v36a y v37) }\end{array}$ & $\begin{array}{l}\text { Variables dependientes (I): opiniones de todas las muestra acerca de: } \\
\text { - La atención que recibe de la sociedad (v12) } \\
\text { - La atención que recibe de las administraciones públicas (v11, v13, } \\
\text { v14 y v15) } \\
\text { - La conveniencia de que las niñas y niños cursen diferentes ciclos } \\
\text { de la educación infantil (v8, v10, v16, v16a, v17, v17a, v18, } \\
\text { v18a, v19, v20 y v21) } \\
\text { - Quién es el mejor proveedor de este servicio (v22) } \\
\text { - Qué problemáticas identifican en esta área (v23 y v24) }\end{array}$ \\
\hline$c^{\circ}$ & \\
\hline $\begin{array}{l}\text { Variables independientes: } \\
\text { - Ideología (v27) } \\
\text { - Clase social (v44) }\end{array}$ & $\begin{array}{l}\text { Variables dependientes (I): opiniones de madres y padres acerca de: } \\
\text { - Prácticas de crianza declaradas (v26) } \\
\text { - Justificación (v26a y v26b) }\end{array}$ \\
\hline
\end{tabular}

2 El término transición se utiliza en sociología para describir cualquier movimiento significativo ya sea en el ámbito escolar (el paso de infantil a primaria, de primaria a secundaria o de bachiller a la universidad, serían también ejemplos válidos) o en otras esferas de la vida de las personas (como la transición escuela-trabajo). En particular, el tránsito del entorno familiar al sistema escolar es un hito importante en la vida de las personas que se vincula además con otros episodios vitales y etapas madurativas como son el desarrollo de la autonomía y la socialización con los otros significantes (Hendry, 1986).

3 Para este trabajo se utiliza P44 estatus socioeconómico como sinónimo de clase social. 
A continuación, presentamos el listado de las variables y las preguntas con las que se corresponden:

\begin{tabular}{ll}
\hline V8 & P8: En su opinión, ¿a qué edad debería empezar la educación obligatoria para niños y niñas? \\
\hline V10 & P10: Vamos a centrarnos ahora en los niños y las niñas más pequeños, es decir, hasta que cumplen 3 años. ¿Con cuál de estas dos frases está Ud. más de acuerdo? (A) Aunque pueda tener al- \\
& gunos beneficios el que asistan a una escuela infantil o guardería, lo mejor para los niños y las niñas menores de 3 años, es que permanezcan en casa (B) Aunque pueda tener algunos beneficios \\
que permanezcan en su casa, lo mejor para los niños y las niñas menores de tres años, es que asistan a una escuela infantil o guardería
\end{tabular}

V1 1 P11: Entre estas dos medidas relativas a la atención y cuidado de los niños y las niñas menores de 3años, ¿̇a cuál cree que las Administraciones Públicas deberían darle prioridad? (A) Facilitar medidas para que las madres y los padres trabajadores puedan tener tiempo para cuidar a sus hiios e hijas (B) Crear centros (escuelas infantiles o guarderías) donde los niños y las niñas estén atendidos (C) Ambos por igual.

V12 P12: En general, ¿icree Ud. que la sociedad en su conjunto le presta mucha, bastante, poca o ninguna atención al tema de la educación de los niños y de las niñas menores de 3 años?

V13 P13: Y las Administraciones Públicas, ¿icree Ud. que le prestan mucha, bastante, poca o ninguna atención al tema de la educación de los niños y de las niñas menores de 3 años?

V14 P14: Siguiendo con las Administraciones Públicas, ¿cree que le prestan a la educación de los niños y de las niñas menores de 3 años más, menos o igual atención que hace 10 años?

V15 P15: En cualquier caso, ¿cree que las Administraciones Públicas le prestan a la educación de los niños y de las niñas menores de 3 años más, menos o igual atención que a la de 3 a 6 años?

V16 P16: Pensando ahora en los niños y las niñas de 3 a 6 años, iconsidera que es necesario que los niños y las niñas de esta edad asistan a una escuela infantil o guardería...?

V16a P16a: ¿Por qué razón lo considera necesario?

V17 P17: Y los niños y las niñas que tienen 1 y 2 años, iconsidera que es necesario que los niños y las niñas de esta edad asistan a una escuela infantil o guardería...?

V17a P17a: ¿Por qué razón lo considera necesario?

V18 P18: Y los niños y las niñas que tienen menos de 1 año, ¿ considera que es necesario que los niños y las niñas de esta edad asistan a una escuela infantil o guardería...?

V18a P18a: ¿Por qué razón lo considera necesario?

V19 P19: A continuación, le voy a mencionar una serie de capacidades que los niños y las niñas pueden haber adquirido al cumplir 3 años. En su opinión, ¿dónde las aprenden mejor, en la familia (con sus padres, sus abuelos, ...) 0 en la escuela?

V20 P20: Para que un niño o una niña menor de 3 años reciba una correcta atención en una escuela infantil o guardería, ¿en qué medida cree que cada una de las siguientes características es: muy, bastante, poco o nada importante?

V21 P21: Y, de todas las características que le acabo de mencionar, ¿cuál sería la más importante para Ud. a la hora de elegir un centro para un niño o una niña menor de 3 años? ¿Y la segunda en importancia?

V22 P22: En conjunto, considerando todos los aspectos anteriores, ¿qué tipo de centro cree Ud. que proporciona una mejor educación infantil, un centro público o un centro privado?

V23 P23: Por su experiencia personal o por lo que tiene entendido, ¿cuál es el principal problema en relación a las escuelas infantiles o guarderías para menores de 3 años? ¿Y el segundo?

V24 P24: De las siguientes medidas que ponen en marcha las Administraciones Públicas, icuál le parece prioritaria para proporcionar atención educativa a los niños y las niñas menores de 3 años?

V25 P25: ¿Tiene Ud. hijos/as?

V25a P25a: ¿Cuántos/as?

V25b P25b: Empezando por el/la menor ¿qué edad/es tiene/n?

V27 P27: Cuando se habla de política se utilizan normalmente las expresiones izquierda y derecha. En esta tarijeta hay una serie de casillas que van de izquierda a derecha. ¿En qué casilla se colocaría Ud.?

V29 P29: Sexo

V30 P30: ¿Cuántos años cumplió Ud. en su último cumpleaños?

V3 l a P31a: ¿Cuáles son los estudios de más alto nivel oficial que Ud. ha cursado (con independencia de que los haya terminado o no)? Por favor, especifique lo más posible, diciéndome el curso en que estaba cuando los terminó (o los interrumpió), y también el nombre que tenían entonces esos estudios.

V34 P34: ¿En cuál de las siguientes situaciones se encuentra Ud. actualmente?

V35 P35: ¿ ¡Y cuál es/era su actual/última ocupación u oficio? Es decir, ¿en qué consiste/tía espećificamente su trabajo? Nos referimos a su ocupación principal: aquélla por la que Ud. (o la persona que aporta más ingresos al hogar) obtiene/nía mayores ingresos.

V36a P36a dice: ¿irabaja/ba Ud. (o la persona que aporta más ingresos al hogar) en la Administración Pública, en una empresa pública, en una empresa privada, en una organización privada sin fines de lucro 0 en el servicio doméstico?

V37 P37 dice: ¿A qué actividad se dedica principalmente la empresa u organización donde Ud. (o la persona que aporta más ingresos al hogar) trabaja/ba?

V38 P38: Sin contarle a Ud., ¿ con cuántas personas convive en su casa habitualmente?

V41 P41: ¿¿Ha nacido Ud. en España?

V43 P43: Condición socioeconómica del INE (+inactivos)

Para estudiar las relaciones arriba mencionadas, se realiza un análisis en varios pasos. En primer lugar, se realiza la descripción de la muestra, a continuación, se presenta un análisis descriptivo que muestra las tendencias generales mediante tablas de contingencia. Estas se complementan con el estadístico Chi-Cuadrado de Pearson que nos permite comprobar qué diferencias son significativas. Este primer análisis nos permite identificar tendencias básicas, así como aquellas áreas del cuestionario en las que centrar el análisis de regresión que completa la parte final del estudio. 


\section{Resultados}

Como se ha indicado al inicio de este artículo, los datos de la encuesta del Centro de Investigaciones Sociológicas (CIS), en el que se basa el presente trabajo, proceden del Barómetro de febrero de 2009 (n. ${ }^{\circ}$ 2788). El estudio es de ámbito nacional, el universo es la población española, de ambos sexos, de 18 años o más. Los puntos de muestreo se repartieron por 238 municipios y 47 provincias y la afijación fue proporcional. La encuesta, mediante entrevista personal en los domicilios, se realizó a 2490 personas entre el 4 y el 10 de febrero de $2009^{4}$.

En relación a las características generales de la muestra, la media de edad ronda los 47 años, el $51 \%$ la constituyen mujeres, el tamaño medio de los hogares encuestados ronda los 3,5 miembros, y la gran mayoría de las personas encuestadas son nacidas en España (97\%). Predominan las y los encuestados con «estudios primarios» (45\%), le siguen aquellos con «formación profesional» (15\%), «estudios secundarios» $(12 \%)$ y «universitarios superiores» $(10 \%)$. Por ocupación el grupo más numeroso lo forman «artesanos y trabajadores cualificados de la industria» (19\%) seguido de «técnicos y profesionales de apoyo» (14\%) y «operadores de maquinaria y montadores» (13\%). El 45\% de la muestra trabajaba en el momento de realizar la encuesta, seguido de un $19 \%$ de jubilados o pensionistas (que anteriormente hubieran trabajado), un $16 \%$ de parados y un $11 \%$ de trabajadoras/es domésticos no remunerados. De los trabajadores por cuenta ajena predominan los empleados en empresas privadas (71\%) seguidos de trabajadores en la administración pública $(20 \%)$.

En la figura 2 se observa la distribución de la variable independiente ideología (v27) agrupada en cinco categorías. En ella se observa que la mayoría de los encuestados se sitúan en posiciones de centro (5-6) y centro izquierda (3-4), siendo más marginal el centro derecha (7-8), la izquierda (1-2) y más aún la derecha (9-10). En los análisis subsiguientes la codificación que se usará es dicotómica izquierda (1-5) y derecha (6-10) excepto en las regresiones que completan el estudio en las que se usa como variable continua.

Figura 2. Distribución de la variable independiente ideología (v27) agrupada en cinco categorías

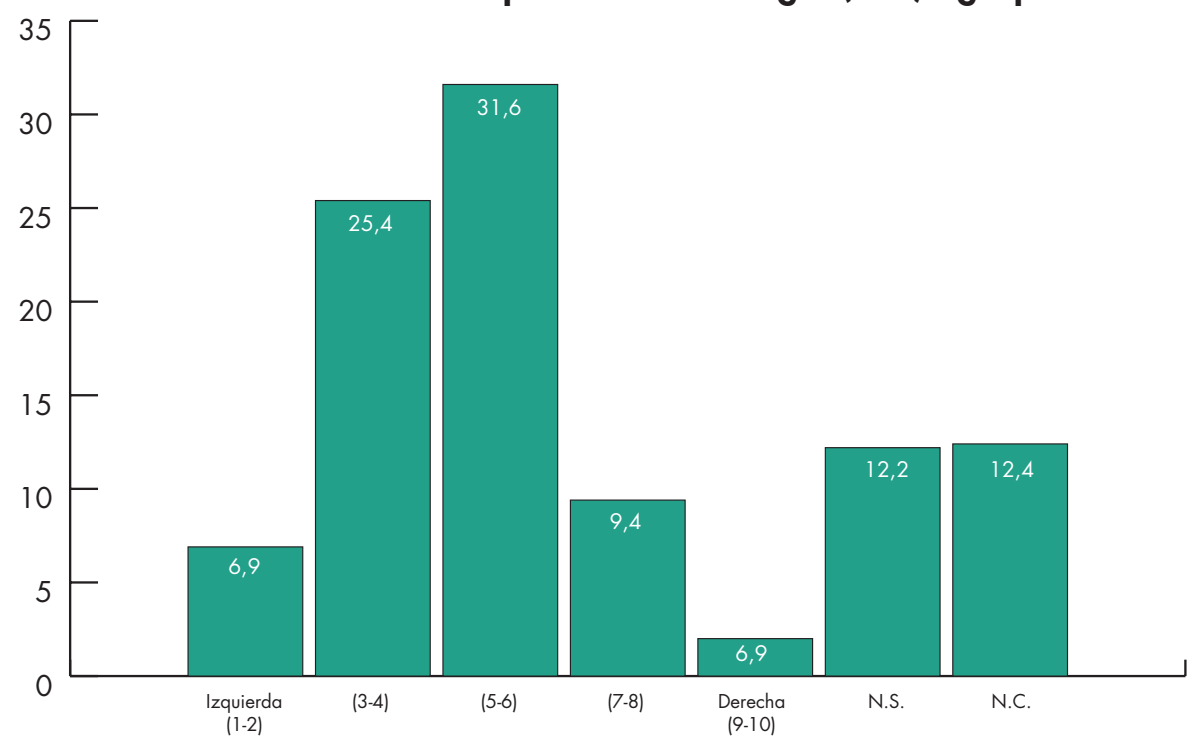

Fuente: elaboración propia a partir del estudio del CIS n. ${ }^{\circ}$ 2788-Barómetro de febrero de 2009.

\footnotetext{
4 Según consta en la ficha técnica, el procedimiento de muestreo reunió las siguientes características: Polietápico, estratificado por conglomerados, con selección de las unidades primarias de muestreo (municipios) y de las unidades secundarias (secciones) de forma aleatoria proporcional, y de las unidades últimas (individuos) por rutas aleatorias y cuotas de sexo y edad. Los estratos se han formado por el cruce de las 17 comunidades autónomas con el tamaño de hábitat, dividido en 7 categorías: menor o igual a 2000 habitantes; de 2001 a 10000; de 10001 a 50000; de 50001 a 100000; de 100001 a 400000 ; de 400001 a 1000000 , y más de 1000000 de habitantes.
} 
Por su parte la figura 3 muestra la distribución de la segunda variable independiente clase social (v44). Predominan en ella los miembros de la «clase obrera cualificada» ${ }^{5}(32,5 \%)$, seguido de los individuos pertenecientes a las «nuevas clases medias» ${ }^{6}(20,1 \%)$ y los de la «clase alta/media-alta» $\rangle^{7}(18,3 \%)$. Cierran la distribución las «viejas clases medias» ${ }^{8}(16,7 \%)$ y la «clase obrera no cualificada» $»^{9}(12,4 \%)$.

\section{Figura 3. Distribución de la variable independiente clase social (v44)}

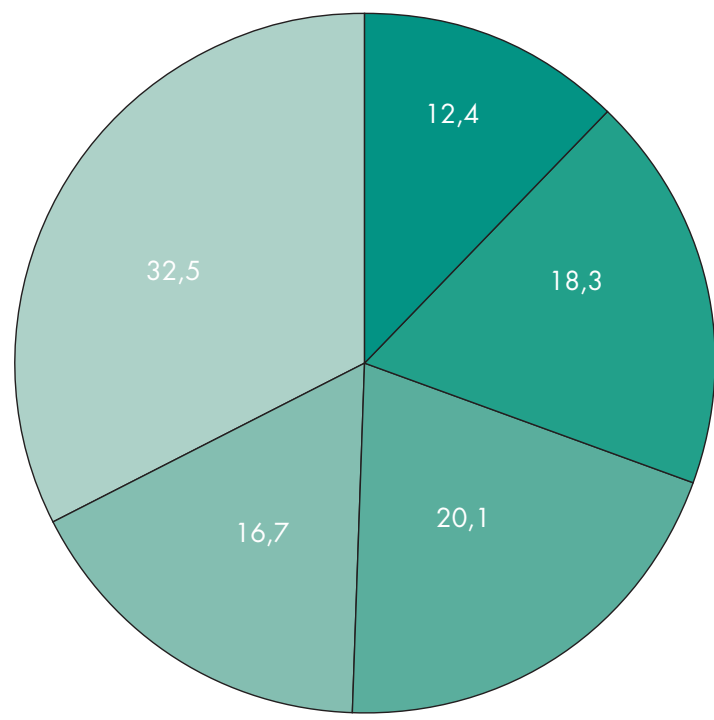

Clase alta/media-alta

Nuevas clases medias

Viejas clases medias

Obreros cualificados

Obreros no cualificados

Fuente: elaboración propia a partir del estudio del CIS n. ${ }^{\circ}$ 2788-Barómetro de febrero de 2009.

Como se indicaba en la introducción, interesa medir la capacidad de las variables ideología (v27) y clase social (v44) para explicar tendencias de respuesta a las siguientes preguntas sobre opiniones respecto de la importancia de la escolarización infantil:

Parte I - Opiniones de toda la muestra acerca de:

a) La atención que recibe de la sociedad (v12) y de las Administraciones Públicas (v11, v13, v14, v15).

b) La conveniencia de que niñas y niños cursen los diferentes ciclos de la educación infantil (v8, v10, v16, v16a, v17, v17a, v18, v18a, v19, v20, v21).

c) Quién es mejor proveedor de este servicio (v22).

d) Qué problemáticas identifican en esta área (v23, v24).

Parte II - Opiniones de madres y padres acerca de:

a) Prácticas de crianza declaradas (v26).

b) Justificación (v26a y v26b).

\footnotetext{
Incluye a manuales cualificados/as, semicualificados/as, capataces/zas y artesanos/as.

Incluye a asalariados/as no manuales.

Incluye a profesionales y técnicos/as, directivos/as y cuadros medios.

Incluye a empresarios/as, autónomos/as y agricultores.

Incluye a obreros/as de la industria y de los servicios, y jornaleros/as.
} 
Parte III - Análisis de regresión (epígrafe «g»)

En las secciones que siguen se desglosa por secciones este análisis de la encuesta.

a) Sobre la importancia de la educación infantil y la atención que recibe

Un primer análisis de las opiniones respecto la importancia que se le otorga a la educación de los niños y niñas menores de 3 años, tanto por la sociedad en general (v12) como por las Administraciones Públicas (v13), nos muestra que se percibe un déficit, más acusado para el caso de las Administraciones Públicas en el que un 72,3\% considera que estas le dan «Poca» o «Ninguna» atención, mientras que cuando hablan de la sociedad en general perciben que esta falta de atención se reduce al 59,2\% (véase figura 4).

Figura 4. Importancia que se le otorga a la educación de los niños y niñas menores de 3 años tanto por la sociedad en general (v12) como por las Administraciones Públicas (v13)

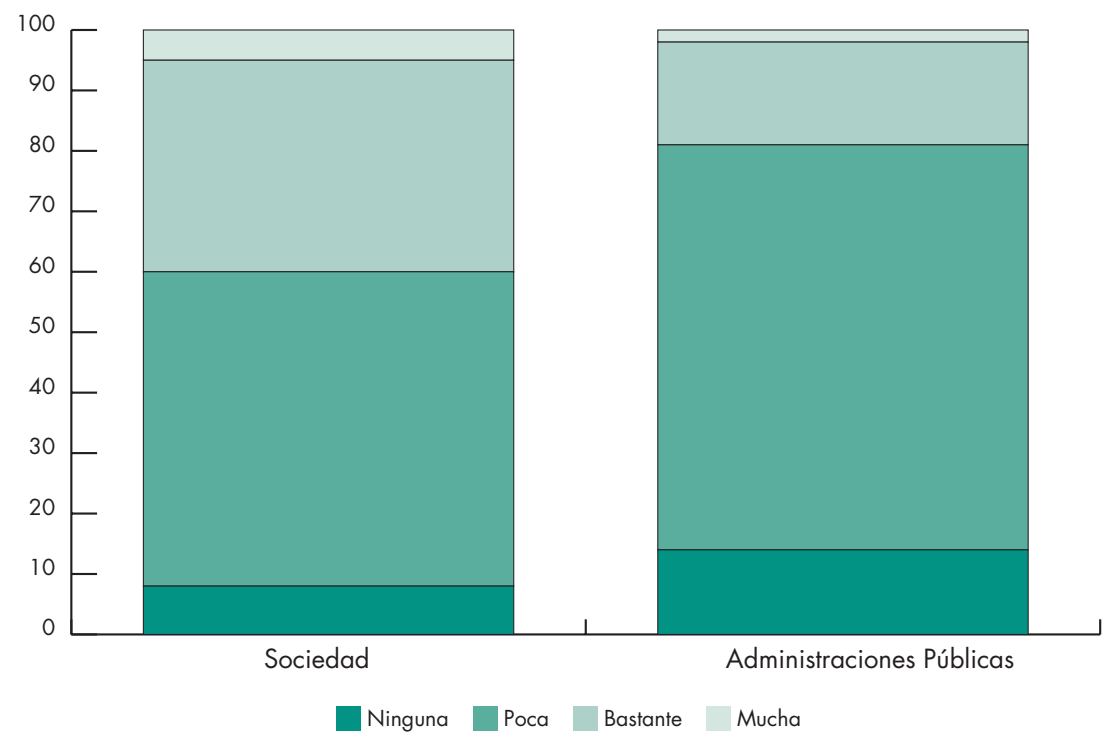

Fuente: elaboración propia a partir del estudio del CIS n. ${ }^{\circ}$ 2788-Barómetro de febrero de 2009.

Como se muestra en las tablas 1 y 2 muestran la distribución por ideología en función de su agrupación como izquierda (1-5) o derecha (6-10). En ellas se aprecia que la gente que se define como de izquierdas tiende a realizar una valoración algo más positiva de la importancia que otorgan a la educación de los niños y niñas menores de 3 años tanto la sociedad en general como las Administraciones Públicas.

\section{Tabla I. Importancia que la sociedad otorga a la educación de los niños y niñas menores de 3 años (v12) en función de la ideología (v27)}

\begin{tabular}{lll}
\hline & IZQUIERDA & DERECHA \\
\hline Mucha & $5,7 \%$ & $4,3 \%$ \\
\hline Bastante & $36,2 \%$ & $31,0 \%$ \\
\hline Poca & $51,1 \%$ & $56,3 \%$ \\
\hline Ninguna & $7,0 \%$ & $8,3 \%$ \\
\hline
\end{tabular}

Pearson Chi-Cuadrado $=6,622 ; \mathrm{gl}=3 ;$ Sig. $=0,085$

Fuente: elaboración propia a partir del estudio del CIS n. ${ }^{\circ}$ 2788-Barómetro de febrero de 2009. 
Tabla II. Importancia que las Administraciones Públicas otorgan a la educación de los niños y niñas menores de 3 años (v13) en función de la ideología (v27)

\begin{tabular}{lll}
\hline & IZQUIERDA & DERECHA \\
\hline Mucha & $3,2 \%$ & $1,8 \%$ \\
\hline Bastante & $26,2 \%$ & $20,0 \%$ \\
\hline Poca & $58,1 \%$ & $62,5 \%$ \\
\hline Ninguna & $12,6 \%$ & $15,8 \%$ \\
\hline
\end{tabular}

Pearson Chi-Cuadrado $=11,935 ; \mathrm{gl}=3$; Sig. $=0,008$

Fuente: elaboración propia a partir del estudio del CIS n. ${ }^{\circ}$ 2788-Barómetro de febrero de 2009.

Por su parte las tablas 3 y 4 muestran que las diferencias de percepción en función de la clase social son relativamente pequeñas, destacando una mejor valoración por las «Viejas clases medias» de la importancia que la sociedad otorga a la educación de primer ciclo de infantil. Estas diferencias no son significativas al valorar la importancia que las Administraciones Públicas otorgan a la educación de los niños y niñas menores de 3 años.

Tabla III. Importancia que la sociedad otorga a la educación de los niños y niñas menores de 3 años (v12) en función de la clase social (v44)

\begin{tabular}{llllll}
\hline & CLASE ALTA/ & NUEVAS CLASES & VIEJAS CLASES & OBREROS & OBREROS \\
& MEDIA-ALTA & MEDIAS & MEDIAS & CUALIFICADOS & NO CUALIFICADOS \\
\hline Mucha & $4,9 \%$ & $5,5 \%$ & $6,6 \%$ & $4,8 \%$ & $8,0 \%$ \\
\hline Bastante & $31,4 \%$ & $33,3 \%$ & $41,6 \%$ & $36,2 \%$ & $28,6 \%$ \\
\hline Poca & $55,5 \%$ & $50,9 \%$ & $45,3 \%$ & $50,9 \%$ & $57,2 \%$ \\
\hline Ninguna & $8,2 \%$ & $10,3 \%$ & $6,6 \%$ & $8,1 \%$ & $6,2 \%$ \\
\hline
\end{tabular}

Pearson Chi-Cuadrado = 25,040; gl = 12; Sig. $=0,015$

Fuente: elaboración propia a partir del estudio del CIS n. ${ }^{\circ}$ 2788-Barómetro de febrero de 2009.

Tabla IV. Importancia que las Administraciones Públicas otorgan a la educación de los niños y niñas menores de 3 años (v13) en función de la clase social (V44)

\begin{tabular}{llllll}
\hline & CLASE ALTA/ & NUEVAS CLASES & VIEJAS CLASES & OBREROS & OBREROS \\
& MEDIA-ALTA & MEDIAS & MEDIAS & $2,9 \%$ & $2,9 \%$ \\
\hline Mucha & $1,9 \%$ & $3,4 \%$ & $25,8 \%$ & $25,0 \%$ & $3,3 \%$ \\
\hline Bastante & $25,0 \%$ & $23,3 \%$ & $60,4 \%$ & $59,5 \%$ & $22,4 \%$ \\
\hline Poca & $58,1 \%$ & $54,6 \%$ & $10,9 \%$ & $12,6 \%$ & $59,9 \%$ \\
\hline Ninguna & $15,0 \%$ & $18,7 \%$ & & $14,3 \%$ \\
\hline
\end{tabular}

Pearson Chi-Cuadrado $=15,173 ; \mathrm{gl}=12 ;$ Sig. $=0,232$

Fuente: elaboración propia a partir del estudio del CIS n. ${ }^{\circ}$ 2788-Barómetro de febrero de 2009.

Las tablas 5 y 6 muestran la distribución de las respuestas, en función de la ideología y la clase social respectivamente a la pregunta sobre cuál cree que debería ser la prioridad de las Administraciones Públicas. Las opciones son bien facilitar el cuidado familiar, bien la escolarización o bien a ambas por igual (v11). Aunque las diferencias se antojan sutiles, si parece haber una ligera tendencia de la «clase alta/media-alta» a preferir que, al hablar de niños menores de tres años, los padres tengan tiempo para cuidar a sus hijas e hijos. Las diferencias entre ideologías sobre este asunto no son significativas. 
Tabla V. Respuesta a la pregunta sobre cuál cree que debería ser la prioridad de las Administraciones Públicas (v1 1) en función de la ideología (v27)

\begin{tabular}{lll}
\hline & IZQUIERDA & DERECHA \\
\hline 1. Que los padres tengan tiempo para cuidar a sus hijos & $53,2 \%$ & $53,4 \%$ \\
\hline 2. Que se creen centros donde los niños sean atendidos & $35,0 \%$ & $37,0 \%$ \\
\hline 3. Ambos por igual (NO LEER) & $11,8 \%$ & $9,6 \%$ \\
\hline
\end{tabular}

Pearson Chi-Cuadrado = 2,064; $\mathrm{gl}=2$; Sig. $=0,356$

Fuente: elaboración propia a partir del estudio del CIS n. ${ }^{\circ}$ 2788-Barómetro de febrero de 2009.

Tabla VI. Respuesta a la pregunta sobre cuál cree que debería ser la prioridad de las Administraciones Públicas (v1 l) en función de la clase social (v44)

\begin{tabular}{llllll}
\hline & $\begin{array}{l}\text { CLASE ALTA/ } \\
\text { MEDIA-ALTA }\end{array}$ & $\begin{array}{l}\text { NUEVAS CLASES } \\
\text { MEDIAS }\end{array}$ & $\begin{array}{l}\text { VIEJAS CLASES } \\
\text { MEDIAS }\end{array}$ & $\begin{array}{l}\text { OBREROS } \\
\text { CUALIFICADOS }\end{array}$ & $\begin{array}{l}\text { OBREROS } \\
\text { NO CUALIFICADOS }\end{array}$ \\
\hline 1. Que los padres tengan tiempo para cuidar a sus hijos & $58,3 \%$ & $53,2 \%$ & $52,6 \%$ & $51,4 \%$ & $54,4 \%$ \\
\hline 2. Que se creen cen-tros donde los niños sean atendidos & $29,4 \%$ & $35,5 \%$ & $34,5 \%$ & $37,8 \%$ & $38,8 \%$ \\
\hline 3. Ambos por igual (NO LEER) & $12,3 \%$ & $11,3 \%$ & $13,0 \%$ & $10,8 \%$ & $6,8 \%$ \\
\hline
\end{tabular}

Pearson Chi-Cuadrado = 16,244; $\mathrm{gl}=8$; Sig. $=0,039$

Fuente: elaboración propia a partir del estudio del CIS n. ${ }^{\circ}$ 2788-Barómetro de febrero de 2009.

La figura 5 y las tablas 7 a 10 ahondan en la cuestión de la atención que las Administraciones Públicas prestan a la educación de los niños y niñas menores de 3 años de manera comparativa con cuanta atención se le prestaba hace diez años (v14) y con cuanta atención se le presta a los del siguiente grupo de edad, esto es 3 a 6 años (v15). En ella se observa cómo la disposición a que las y los menores en edad pre-obligatoria sean escolarizados se incrementa drásticamente conforme se incrementa su edad. También se aprecia cómo, aunque siendo pequeñas las diferencias, los encuestados con ideología de izquierdas tendían a valorar más positivamente la evolución de la atención a la infancia en los diez años trascurridos entre 2000 y 2009.

Figura 5. Atención que las Administraciones Públicas prestan a la educación de los niños y niñas menores de 3 años en relación a hace diez años (v14) y con la que prestan a niños y niñas de 3 a 6 años (v15)

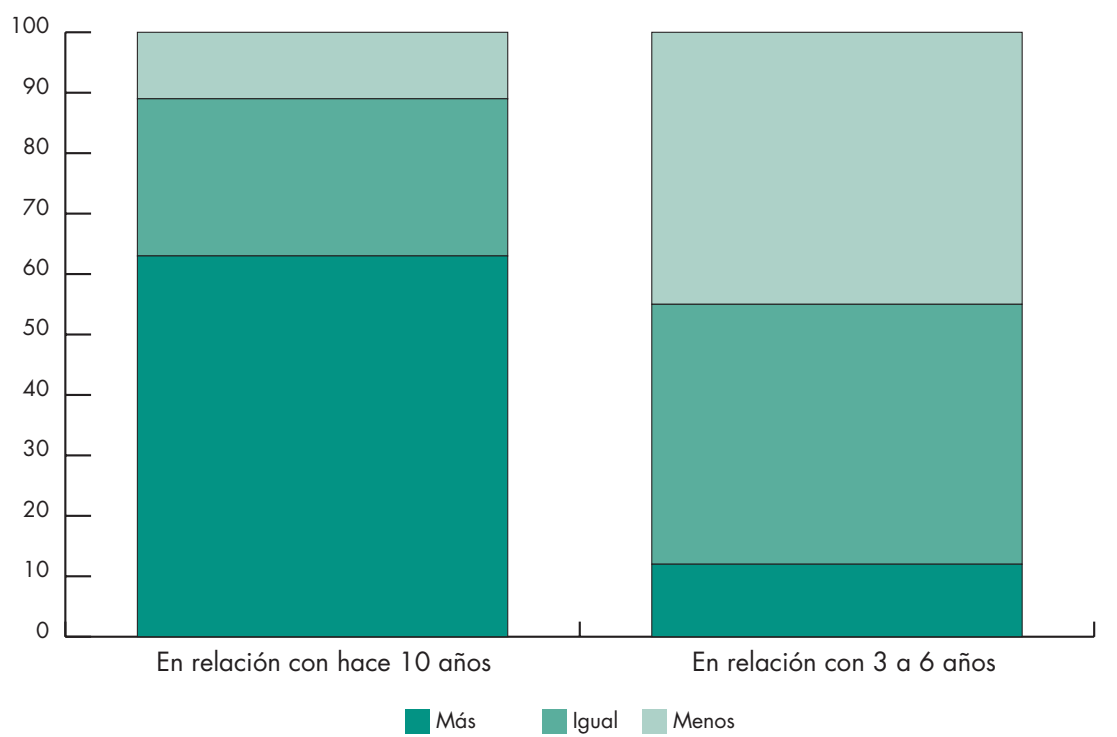

Fuente: elaboración propia a partir del estudio del CIS n. ${ }^{\circ}$ 2788-Barómetro de febrero de 2009. 
Tabla VII. Comparativa de la atención que las Administraciones Públicas prestan a la educación de los niños y niñas menores de 3 años con hace diez años (v14) en función de la ideología (v27)

\begin{tabular}{lll}
\hline & IZQUIERDA & DERECHA \\
\hline Más & $66,7 \%$ & $60,9 \%$ \\
\hline Menos & $9,2 \%$ & $15,3 \%$ \\
\hline Igual & $24,1 \%$ & $23,7 \%$ \\
\hline
\end{tabular}

Pearson Chi-Cuadrado = 13,420; $\mathrm{gl}=2$; Sig. $=0,001$

Fuente: elaboración propia a partir del estudio del CIS n. ${ }^{\circ}$ 2788-Barómetro de febrero de 2009.

Tabla VIII. Comparativa de la atención que las Administraciones Públicas prestan a la educación de los niños y niñas menores de 3 años con la que prestan a niños

y niñas de 3 a 6 años ( $v 15$ ) en función de la ideología (v27)

\begin{tabular}{lll}
\hline & IZQUIERDA & DERECHA \\
\hline Más & $13,7 \%$ & $12,0 \%$ \\
\hline Menos & $47,4 \%$ & $47,9 \%$ \\
\hline Igual & $38,8 \%$ & $40,1 \%$ \\
\hline
\end{tabular}

Pearson Chi-Cuadrado = 0,848; $\mathrm{gl}=2$; Sig. $=0,654$

Fuente: elaboración propia a partir del estudio del CIS n. ${ }^{\circ}$ 2788-Barómetro de febrero de 2009.

Tabla IX. Comparativa de la atención que las Administraciones Públicas prestan a la educación de los niños y niñas menores de 3 años con hace diez años (v14) en función de la clase social (v44)

\begin{tabular}{llllll}
\hline & CLASE ALTA/ & NUEVAS CLASES & VIEJAS CLASES & OBREROS & OBREROS \\
& MEDIA-ALTA & MEDIAS & MEDIAS & CUALIFICADOS & NO CUALIFICADOS \\
\hline Más & $65,9 \%$ & $68,9 \%$ & $60,9 \%$ & $60,1 \%$ & $63,7 \%$ \\
\hline Igual & $22,7 \%$ & $22,4 \%$ & $27,7 \%$ & $26,9 \%$ & $24,2 \%$ \\
\hline Menos & $11,4 \%$ & $8,7 \%$ & $11,4 \%$ & $12,9 \%$ & $12,1 \%$ \\
\hline
\end{tabular}

Pearson Chi-Cuadrado = 12,154; gl = 8; Sig. $=0,144$

Fuente: elaboración propia a partir del estudio del CIS n. ${ }^{\circ}$ 2788-Barómetro de febrero de 2009.

Tabla X. Comparativa de la atención que las Administraciones Públicas prestan a la educación de los niños y niñas menores de 3 años con la que prestan a niños y niñas de 3 a 6 años (v15) en función de la clase social (v44)

\begin{tabular}{llllll}
\hline & CLASE ALTA/ & NUEVAS CLASES & VIEJAS CLASES & OBREROS & OBREROS \\
& MEDIA-ALTA & MEDIAS & MEDIAS & $\begin{array}{l}\text { CUALIFICADOS } \\
\text { NO CUALIFICADOS }\end{array}$ \\
\hline Más & $10,9 \%$ & $13,5 \%$ & $14,3 \%$ & $12,5 \%$ & $17,9 \%$ \\
\hline Igual & $28,8 \%$ & $36,8 \%$ & $40,9 \%$ & $48,2 \%$ & $40,9 \%$ \\
\hline Menos & $60,4 \%$ & $49,8 \%$ & $44,8 \%$ & $39,3 \%$ & $41,3 \%$ \\
\hline
\end{tabular}

Pearson Chi-Cuadrado = 55,580; $\mathrm{gl}=8$; Sig. $=0,000$

Fuente: elaboración propia a partir del estudio del CIS n. ${ }^{\circ}$ 2788-Barómetro de febrero de 2009.

\section{b) Sobre la conveniencia de la escolarización en educación infantil}

Las tablas 11 a 14 nos muestran las repuestas a las preguntas sobre ¿a qué edad piensan llas y los encuestados] que debería empezar la educación obligatoria para niños y niñas? (v8) y a qué edad creen que empieza la educación obligatoria para niños y niñas en la actualidad (v9), ambas en función de la ideología (v27) y la clase social (v44). Los datos muestran una extendida confusión entre edad de gratuidad (3 años de edad) y edad obligatoria (6 años de edad), esta confusión es algo más pronunciada entre las Viejas clases medias, los Obreros cualificados, y los Obreros no cualificados. Las diferencias por ideología no son significativas. 
Tabla XI. Comparativa de la atención que las Administraciones Públicas prestan a la educación de los niños y niñas menores de 3 años con hace diez años (v14) en función de la ideología (v27)

\begin{tabular}{lll}
\hline & IZQUIERDA & DERECHA \\
\hline Antes de cumplir los 3 años & $15,2 \%$ & $13,0 \%$ \\
\hline A los 3 años & $54,1 \%$ & $53,1 \%$ \\
\hline A los 4 años & $15,5 \%$ & $18,8 \%$ \\
\hline A los 5 años & $9,2 \%$ & $9,1 \%$ \\
\hline A los 6 o más años & $6,0 \%$ & $6,0 \%$ \\
\hline
\end{tabular}

Pearson Chi-Cuadrado $=3,730 ; \mathrm{gl}=4$; Sig. $=0,444$

Fuente: elaboración propia a partir del estudio del CIS n. ${ }^{\circ}$ 2788-Barómetro de febrero de 2009.

Tabla XII. Respuesta a la pregunta sobre a qué edad creen que empieza la educación obligatoria para niños y niñas (v9) en función de la ideología (v27)

Pearson Chi-Cuadrado = 2,349; gl $=4$; Sig. $=0,672$

\begin{tabular}{lll}
\hline & IZQUIERDA & DERECHA \\
\hline Antes de cumplir los 3 años & $5,6 \%$ & $5,0 \%$ \\
\hline$A \operatorname{los} 3$ años & $55,3 \%$ & $58,5 \%$ \\
\hline A los 4 años & $13,4 \%$ & $11,3 \%$ \\
\hline$A \operatorname{los} 5$ años & $9,0 \%$ & $7,9 \%$ \\
\hline A los 6 o más años & $16,7 \%$ & $17,2 \%$ \\
\hline
\end{tabular}

Fuente: elaboración propia a partir del estudio del CIS n. ${ }^{\circ}$ 2788-Barómetro de febrero de 2009.

Tabla XIII. Respuesta a la pregunta sobre a qué edad piensan que debería empezar la educación obligatoria para niños y niñas (v8) en función de la clase social (v44)

\begin{tabular}{llllll}
\hline & $\begin{array}{l}\text { CLASE ALTA/ } \\
\text { MEDIA-ALTA }\end{array}$ & $\begin{array}{l}\text { NUEVAS CLASES } \\
\text { MEDIAS }\end{array}$ & $\begin{array}{l}\text { VIEJAS CLASES } \\
\text { MEDIAS }\end{array}$ & $\begin{array}{l}\text { OBREROS } \\
\text { CUALIFICADOS }\end{array}$ & $\begin{array}{l}\text { OBREROS } \\
\text { NO CUALIFICADOS }\end{array}$ \\
\hline Antes de cumplir los 3 años & $15,2 \%$ & $14,8 \%$ & $11,0 \%$ & $14,5 \%$ & $12,8 \%$ \\
\hline$A \operatorname{los} 3$ años & $56,5 \%$ & $56,2 \%$ & $53,7 \%$ & $52,0 \%$ & $55,2 \%$ \\
\hline A los 4 años & $11,1 \%$ & $15,2 \%$ & $20,9 \%$ & $18,8 \%$ & $15,2 \%$ \\
\hline$A \operatorname{los} 5$ años & $9,4 \%$ & $8,7 \%$ & $9,1 \%$ & $8,4 \%$ & $13,1 \%$ \\
\hline$A \operatorname{los} 6$ más años & $7,8 \%$ & $5,1 \%$ & $5,3 \%$ & $6,4 \%$ & $3,8 \%$ \\
\hline
\end{tabular}

Pearson Chi-Cuadrado = 31,652; gl = 16; Sig. $=0,011$

Fuente: elaboración propia a partir del estudio del CIS n. ${ }^{\circ}$ 2788-Barómetro de febrero de 2009.

Tabla XIV. Respuesta a la pregunta sobre a qué edad creen que empieza la educación obligatoria para niños y niñas (v9) en función de la clase social (v44)

\begin{tabular}{llllll}
\hline & $\begin{array}{l}\text { CLASE ALTA/ } \\
\text { MEDIA-ALTA }\end{array}$ & $\begin{array}{l}\text { NUEVAS CLASES } \\
\text { MEDIAS }\end{array}$ & $\begin{array}{l}\text { VIEJAS CLASES } \\
\text { MEDIAS }\end{array}$ & $\begin{array}{l}\text { OBREROS } \\
\text { CUALIFICADOS }\end{array}$ & $\begin{array}{l}\text { OBREROS } \\
\text { NO CUALIFICADOS }\end{array}$ \\
\hline Antes de cumplir los 3 años & $4,7 \%$ & $4,5 \%$ & $6,0 \%$ & $6,3 \%$ & $6,3 \%$ \\
\hline A los 3 años & $53,4 \%$ & $53,5 \%$ & $62,5 \%$ & $59,1 \%$ & $58,4 \%$ \\
\hline A los 4 años & $11,7 \%$ & $12,6 \%$ & $15,5 \%$ & $14,1 \%$ & $10,2 \%$ \\
\hline A los 5 años & $7,5 \%$ & $6,9 \%$ & $6,0 \%$ & $8,7 \%$ & $11,8 \%$ \\
\hline A los 6 mós años & $22,8 \%$ & $22,4 \%$ & $10,1 \%$ & $11,8 \%$ & $13,3 \%$ \\
\hline
\end{tabular}

Pearson Chi-Cuadrado = 54,205; gl = 16; Sig. $=0,000$

Fuente: elaboración propia a partir del estudio del CIS n. ${ }^{\circ}$ 2788-Barómetro de febrero de 2009. 
Las tablas 15 y 16 muestra la respuesta a ¿con cuál de dos frases ofrecidas está más de acuerdo en relación con los niños y las niñas de menos de 3 años? (v10) en función de la ideología (v27) y la clase social (v44). Las posibles respuestas decían: «(A) Aunque pueda tener algunos beneficios el que asistan a una escuela infantil o guardería, lo mejor para los niños y las niñas menores de 3 años, es que permanezcan en casa» $\mathrm{y}$ «(B) Aunque pueda tener algunos beneficios que permanezcan en su casa, lo mejor para los niños y las niñas menores de tres años, es que asistan a una escuela infantil o guardería». En ella predomina la opción segunda, que asistan a un centro de educación infantil de primer ciclo, pero ni las diferencias por ideología ni por clase social son significativas.

Tabla XV. Respuesta a la pregunta sobre si creen que es mejor dejar a los niños y las niñas de menos de 3 años en casa o llevarlos a una escuela infantil - guardería (v10) en función de la clase social (V44)

\begin{tabular}{lll}
\hline & IZQUIERDA & DERECHA \\
\hline (A) Que permanezcan en casa & $43,2 \%$ & $39,7 \%$ \\
\hline (B) Que asistan a una escuela infantil o guardería & $56,8 \%$ & $60,3 \%$ \\
\hline
\end{tabular}

Pearson Chi-Cuadrado = 1,853; gl = 1; Sig. $=0,173$

Fuente: elaboración propia a partir del estudio del CIS n. ${ }^{\circ}$ 2788-Barómetro de febrero de 2009.

Tabla XVI. Respuesta a la pregunta sobre si creen que es mejor dejar a los niños y las niñas de menos de 3 años en casa o llevarlos a una escuela infantil o guardería (v10) en función de la clase social (v44)

\begin{tabular}{llllll}
\hline & CLASE ALTA/ & NUEVAS CLASES & VIEJAS CLASES \\
& MEDIA-ALTA & MEDIAS & OBREROS & OBREROS \\
CUALIFICADOS & NO CUALIFICADOS \\
\hline Que permanezcan en casa & $39,4 \%$ & $41,6 \%$ & $47,4 \%$ & $47,4 \%$ & $45,6 \%$ \\
\hline Que asistan a una escuela infantil o guardería & $60,6 \%$ & $58,4 \%$ & $52,6 \%$ & $56,1 \%$ & $54,4 \%$ \\
\hline
\end{tabular}

Pearson Chi-Cuadrado $=6,562 ; \mathrm{gl}=4$; Sig. $=0,161$

Fuente: elaboración propia a partir del estudio del CIS n. ${ }^{\circ}$ 2788-Barómetro de febrero de 2009.

Por su parte, la tabla 17 muestra el grado de acuerdo con que niños y niñas de diferentes edades se escolaricen (v16, v17, v18). En ella se observa cómo la disposición a que las y los menores en edad pre-obligatoria sean escolarizados se incrementa drásticamente conforme se incrementa su edad partiendo de solo un 5,5\% cuando hablamos de escolarizar en todos los casos a menores de un año, hasta el 79,5\% cuando hablamos de escolarizar en todos los casos a niñas y niños de entre 3 y 6 años de edad.

Tabla XVII. Respuesta a la pregunta de si niños y niñas de diferentes grupos de edad deberían o no escolarizarse $(\mathrm{v} 16, \mathrm{v} 17, \mathrm{v} 18)$

\begin{tabular}{llll}
\hline & 3 A 6 AÑOS & 1 Y 2 AÑOS & MENOS DE 1 AÑO \\
\hline 1. En todos los casos & $79,5 \%$ & $16,6 \%$ & $5,5 \%$ \\
\hline 2. En algunos casos & $17,5 \%$ & $50,1 \%$ & $27,6 \%$ \\
\hline 3. En ningún caso & $3,0 \%$ & $33,3 \%$ & $66,9 \%$ \\
\hline
\end{tabular}

Fuente: elaboración propia a partir del estudio del CIS n. ${ }^{\circ}$ 2788-Barómetro de febrero de 2009.

Continuando con este análisis, las tablas de la 18 a la 23 muestran las diferencias de opinión, en función de la ideología y la clase social, acerca de porque consideran que niños y niñas de tres diferentes grupos de edades (de 3 a 6 años; de 1 y 2 años; y de menos de 1 año) deberían 
escolarizarse (v16a, v17a, v18a). En ellas se observa una tendencia consistente, e independiente del grupo de edad de los niños y niñas, a justificar su conveniencia por razones de conciliación ligeramente mayor en las clases sociales menos favorecidas, así como en quienes se identifican con posiciones más conservadoras o de derechas. Y, por el contrario, mayor tendencia a justificar esa misma conveniencia de escolarización, pero por razones de reducción de las diferencias culturales y sociales, a aquellas clases sociales más favorecidas, y a aquellos quienes se consideran más progresista o de izquierdas.

Tabla XVIII. Respuesta a la pregunta de porque consideran que niños y niñas de 3 a 6 años deberían escolarizarse (v16a), en función de la ideología (v27)

\begin{tabular}{lll}
\hline & IZQUIERDA & DERECHA \\
\hline 1. Porque tendrían resuelto el cuidado de los niños y niñas & $25,1 \%$ & $39,7 \%$ \\
\hline 2. Porque la escuela reducen las diferencias sociales & $10,2 \%$ & $7,9 \%$ \\
\hline 3. Porque la escuela facilita la convivencia & $41,0 \%$ & $34,8 \%$ \\
\hline 4. Porque los niños comienzan antes a adquirir conocimientos & $22,4 \%$ & $28,1 \%$ \\
\hline 5. Otra razón & $1,2 \%$ & $0,8 \%$ \\
\hline
\end{tabular}

Pearson Chi-Cuadrado = 12,731; gl = 4; Sig. $=0,013$

Fuente: elaboración propia a partir del estudio del CIS n. ${ }^{\circ}$ 2788-Barómetro de febrero de 2009.

Tabla XIX. Respuesta a la pregunta de porque consideran que niños y niñas de 3 a 6 años deberían escolarizarse (v16a), en función de la clase social (v44)

\begin{tabular}{|c|c|c|c|c|c|}
\hline & $\begin{array}{l}\text { CLASE ALTA/ } \\
\text { MEDIA-ALTA }\end{array}$ & $\begin{array}{l}\text { NUEVAS CLASES } \\
\text { MEDIAS }\end{array}$ & $\begin{array}{l}\text { VIEJAS CLASES } \\
\text { MEDIAS }\end{array}$ & $\begin{array}{l}\text { OBREROS } \\
\text { CUALIFICADOS }\end{array}$ & $\begin{array}{l}\text { OBREROS } \\
\text { NO CUALIFICADOS }\end{array}$ \\
\hline 1. Porque tendrían resuelto el cuidado de los niños y niñas & $23,4 \%$ & $24,7 \%$ & $27,8 \%$ & $27,6 \%$ & $31,5 \%$ \\
\hline 2. Porque la escuela reducen las diferencias sociales & $12,6 \%$ & $9,7 \%$ & $6,1 \%$ & $6,3 \%$ & $5,9 \%$ \\
\hline 3. Porque la escuela facilita la convivencia & $38,6 \%$ & $41,5 \%$ & $39,4 \%$ & $39,6 \%$ & $38,5 \%$ \\
\hline 4. Porque los niños comienzan antes a adquirir conocimientos & $23,9 \%$ & $23,7 \%$ & $26,4 \%$ & $25,3 \%$ & $22,0 \%$ \\
\hline 5. Otra razón & $1,4 \%$ & $0,4 \%$ & $0,3 \%$ & $1,3 \%$ & $2,1 \%$ \\
\hline
\end{tabular}

Pearson Chi-Cuadrado $=33,565 ; \mathrm{gl}=16$; Sig. $=0,006$

Fuente: elaboración propia a partir del estudio del CIS n. ${ }^{\circ}$ 2788-Barómetro de febrero de 2009.

Tabla XX. Respuesta a la pregunta de porque consideran que niños y niñas de 1 y 2 años deberían escolarizarse (v17a), en función de la ideología (v27)

\begin{tabular}{lll}
\hline & IZQUIERDA & DERECHA \\
\hline 1. Porque tendrían resuelto el cuidado de los niños y niñas & $62,7 \%$ & $67,1 \%$ \\
\hline 2. Porque la escuela reducen las diferencias sociales & $4,8 \%$ & $3,7 \%$ \\
\hline 3 Porque la escuela facilita la convi-vencia & $23,0 \%$ & $22,4 \%$ \\
\hline 4 Porque los niños comienzan antes a adquirir conocimientos & $9,0 \%$ & $6,5 \%$ \\
\hline 5. Otra razón & $0,6 \%$ & $0,3 \%$ \\
\hline
\end{tabular}

Pearson Chi-Cuadrado $=3,757 ; \mathrm{gl}=4$; Sig. $=0,440$

Fuente: elaboración propia a partir del estudio del CIS n. ${ }^{\circ}$ 2788-Barómetro de febrero de 2009. 
Tabla XXI. Respuesta a la pregunta de porque consideran que niños y niñas de 1 y 2 años deberían escolarizarse (v17a), en función de la clase social (v44)

\begin{tabular}{llllll}
\hline & $\begin{array}{l}\text { CLASE ALTA/ } \\
\text { MEDIA-ALTA }\end{array}$ & $\begin{array}{l}\text { NUEVAS CLASES } \\
\text { MEDIAS }\end{array}$ & $\begin{array}{l}\text { VIEJAS CLASES } \\
\text { MEDIAS }\end{array}$ & $\begin{array}{l}\text { OBREROS } \\
\text { CUALIFICADOS }\end{array}$ & $\begin{array}{l}\text { OBREROS } \\
\text { NO CUALIFICADOS }\end{array}$ \\
\hline 1. Porque tendrían resuelto el cuidado de los niños y niñas & $55,7 \%$ & $68,6 \%$ & $67,9 \%$ & $65,5 \%$ & $70,8 \%$ \\
\hline 2. Porque la escuela reducen las diferencias sociales & $6,8 \%$ & $2,8 \%$ & $3,4 \%$ & $2,9 \%$ & $3,1 \%$ \\
\hline 3. Porque la escuela facilita la convivencia & $28,0 \%$ & $20,3 \%$ & $20,3 \%$ & $23,1 \%$ & $16,9 \%$ \\
\hline 4. Porque los niños comienzan antes a adquirir conocimientos & $8,8 \%$ & $8,3 \%$ & $8,0 \%$ & $7,9 \%$ & $9,2 \%$ \\
\hline 5. Otra razón & $0,7 \%$ & & $0,4 \%$ & $0,6 \%$ & \\
\hline
\end{tabular}

Pearson Chi-Cuadrado $=27,751 ; \mathrm{gl}=16$; Sig. $=0,034$

Fuente: elaboración propia a partir del estudio del CIS n. ${ }^{\circ}$ 2788-Barómetro de febrero de 2009.

Tabla XXII. Respuesta a la pregunta de porque consideran que niños y niñas de menos de 1 año deberían escolarizarse (v18a), en función de la ideología (v27)

\begin{tabular}{lll}
\hline & IZQUIERDA & DERECHA \\
\hline 1. Porque tendrían resuelto el cuidado de los niños y niñas & $78,4 \%$ & $87,4 \%$ \\
\hline 2. Porque la escuela reducen las diferencias sociales & $2,0 \%$ & $1,2 \%$ \\
\hline 3 Porque la escuela facilita la convi-vencia & $11,6 \%$ & $8,4 \%$ \\
\hline 4 Porque los niños comienzan antes a adquirir conocimientos & $7,3 \%$ & $2,4 \%$ \\
\hline 5. Otra razón & $0,7 \%$ & $0,6 \%$ \\
\hline
\end{tabular}

Pearson Chi-Cuadrado $=7,817 ; \mathrm{gl}=4$; Sig. $=0,099$

Fuente: elaboración propia a partir del estudio del CIS n. ${ }^{\circ}$ 2788-Barómetro de febrero de 2009.

Tabla XXIII. Respuesta a la pregunta de porque consideran que niños y niñas de menos de 1 año deberían escolarizarse (v18a), en función de la clase social (v44)

\begin{tabular}{llllll}
\hline & $\begin{array}{l}\text { CLASE ALTA/ } \\
\text { MEDIA-ALTA }\end{array}$ & $\begin{array}{l}\text { NUEVAS CLASES } \\
\text { MEDIAS }\end{array}$ & $\begin{array}{l}\text { VIEJAS CLASES } \\
\text { MEDIAS }\end{array}$ & $\begin{array}{l}\text { OBREROS } \\
\text { CUALIFICADOS }\end{array}$ & $\begin{array}{l}\text { OBREROS } \\
\text { NO CUALIFICADOS }\end{array}$ \\
\hline 1. Porque tendrían resuelto el cuidado de los niños y niñas & $76,9 \%$ & $85,4 \%$ & $80,5 \%$ & $82,2 \%$ & $82,8 \%$ \\
\hline 2. Porque la escuela reducen las differencias sociales & $4,4 \%$ & $0,6 \%$ & $2,3 \%$ & $0,9 \%$ & $8,1 \%$ \\
\hline 3. Porque la escuela facilita la convivencia & $9,4 \%$ & $7,6 \%$ & $13,3 \%$ & $12,8 \%$ & $8,1 \%$ \\
\hline 4. Porque los niños comienzan antes a adquirir conocimientos & $8,8 \%$ & $5,7 \%$ & $3,9 \%$ & $3,7 \%$ & $1,0 \%$ \\
\hline 5. Otra razón & $0,6 \%$ & $0,6 \%$ & & $0,5 \%$ & \\
\hline
\end{tabular}

Pearson Chi-Cuadrado = 22,365; gl = 16; Sig. $=0,132$

Fuente: elaboración propia a partir del estudio del CIS n. ${ }^{\circ}$ 2788-Barómetro de febrero de 2009.

En las tablas 24 y 25 se aprecia que existe un grado muy notable de acuerdo en cuanto a que capacidades los niños y niñas de hasta tres años aprenden mejor en casa (expresar necesidades, autonomía alimentación e higiene, o psicomotricidad) y cuales en la escuela (relación y comunicación, observar y explorar el entorno, hábitos y rutinas). Entre ellas destacan «expresar necesidades» y «psicomotricidad» como las tareas que mejor se trabajan en casa frente a la escuela, predominando en esta última la «relación y comunicación» función socializadora, ya aludida en otras preguntas del cuestionario. No obstante, las diferencias no son significativas excepto en el caso de las diferencias por clase social respecto de la «autonomía, alimentación e higiene». 
Tabla XXIV. Respuesta a la pregunta hasta los 3 años de edad, ¿dónde aprenden mejor en familia $(-1)$ o en la escuela (+1) ciertas capacidades (v19)? en función de la ideología (v27)

\begin{tabular}{llll}
\hline & IZQUIERDA & DERECHA & SIG. \\
\hline Expresar necesidades & $-0,3507$ & $-0,3522$ & 0,9730 \\
\hline Autonomía alimentación e higiene & $-0,1649$ & $-0,1512$ & 0,7703 \\
\hline Psicomotricidad & $-0,3226$ & $-0,3638$ & 0,3398 \\
\hline Relación y comunicación & 0,5715 & 0,5932 & 0,5584 \\
\hline Observar y explorar el entorno & 0,2397 & 0,1727 & 0,1150 \\
\hline Hábitos y rutinas & 0,2134 & 0,2180 & 0,9155 \\
\hline
\end{tabular}

Fuente: elaboración propia a partir del estudio del CIS n. ${ }^{\circ}$ 2788-Barómetro de febrero de 2009.

Tabla XXV. Respuesta a la pregunta hasta los 3 años de edad, ¿dónde aprenden mejor en familia $(-1)$ o en la escuela (+1) ciertas capacidades (v19)? en función de la clase social (v44)

\begin{tabular}{lllllll}
\hline & $\begin{array}{l}\text { CLASE ALTA/ } \\
\text { MEDIA-ALTA }\end{array}$ & $\begin{array}{l}\text { NUEVAS CLASES } \\
\text { MEDIAS }\end{array}$ & $\begin{array}{l}\text { VIEJAS CLASES } \\
\text { MEDIAS }\end{array}$ & $\begin{array}{l}\text { OBREROS } \\
\text { CUALIFICADOS }\end{array}$ & $\begin{array}{l}\text { OBREROS } \\
\text { NO CUALIFICADOS }\end{array}$ & SIG. \\
\hline Expresar necesidades & $-0,350$ & $-0,345$ & $-0,426$ & $-0,342$ & $-0,320$ & 0,474 \\
\hline Autonomía alimentación e higiene & $-0,189$ & $-0,132$ & $-0,309$ & $-0,170$ & $-0,126$ & 0,035 \\
\hline Psicomotricidad & $-0,310$ & $-0,317$ & $-0,420$ & $-0,322$ & $-0,347$ & 0,306 \\
\hline Relación y comunicación & 0,551 & 0,580 & 0,513 & 0,571 & 0,532 & 0,652 \\
\hline Observar y explorar el entorno & 0,239 & 0,198 & 0,146 & 0,185 & 0,211 & 0,590 \\
\hline Rutinas y normas & 0,226 & 0,178 & 0,137 & 0,209 & 0,236 & 0,499 \\
\hline
\end{tabular}

Fuente: elaboración propia a partir del estudio del CIS n. ${ }^{\circ}$ 2788-Barómetro de febrero de 2009.

Las tablas 26 y 27 muestra la repuesta a la pregunta (v20) sobre qué importancia le otorga a una serie de características, de la escuela infantil o guardería, para que un niño o una niña menor de 3 años reciban una correcta atención. En ella se aprecia que existen un alto grado de coincidencia en la importancia que le otorgan a las diferentes cuestiones planteadas siendo las más relevantes «que se les dé una alimentación correcta y equilibrada» y «que el personal sea cariñoso y afectuoso» seguidas de «que los locales sean amplios y en buenas condiciones» y de «que el personal tenga un título oficial» y «que haya un programa educativo específico»". No existe significación en las diferencias entre ideologías, excepto por «que el personal tenga un título oficial», ligeramente más valorado por los de derechas. Y existen ligeras variaciones entre clases sociales en relación a «que haya un programa educativo específico» y «que haya pocos niños en el aula» (más valorado por Clase alta/ media-alta y Nuevas clases medias), y en relación a «que tenga servicio de transporte» (más valorado por las Viejas clases medias, los Obreros cualificados y los Obreros no cualificados).

Tabla XXVI. Respuesta a la pregunta ¿en qué medida cree que cada una de las siguientes características es: muy, bastante, poco o nada importante para que un niño o una niña menor de 3 años reciba una correcta atención en una escuela infantil o guardería? (v20) en función de la ideología (v27)

\begin{tabular}{llll}
\hline & IZQUIERDA & DERECHA & SIG. \\
\hline Que el personal tenga un título oficial & 3,640 & 3,716 & 0,005 \\
\hline Que los locales sean amplios y en buenas condiciones & 3,702 & 3,687 & 0,548 \\
\hline Que haya pocos niños en el aula & 3,477 & 3,512 & 0,259 \\
\hline Que haya un programa educativo específico & 3,598 & 3,597 & 0,989 \\
\hline
\end{tabular}




\begin{tabular}{llll}
\hline & IZQUIERDA & DERECHA & SIG. \\
\hline Que el personal sea cariñoso y afectuoso & 3,777 & 3,771 & 0,785 \\
\hline Que ofrezca flexibilidad de horarios & 3,551 & 3,540 & 0,713 \\
\hline Que se les dé una alimentación correcta y equilibrada & 3,794 & 3,785 & 0,712 \\
\hline Que ofrezca la enseñanza de idiomas & 3,056 & 3,006 & 0,270 \\
\hline Que tenga servicio de transporte & 3,256 & 3,218 & 0,341
\end{tabular}

Fuente: elaboración propia a partir del estudio del CIS n. ${ }^{\circ}$ 2788-Barómetro de febrero de 2009.

Tabla XXVII. Respuesta a la pregunta ¿en qué medida cree que cada una de las siguientes características es: muy, bastante, poco o nada importante para que un niño

o una niña menor de 3 años reciba una correcta atención en una escuela infantil o guardería? en función de la clase social (v44)

\begin{tabular}{lllllll}
\hline & $\begin{array}{l}\text { CLASE ALTA/ } \\
\text { MEDIA-ALTA }\end{array}$ & $\begin{array}{l}\text { NUEVAS CLASES } \\
\text { MEDIAS }\end{array}$ & $\begin{array}{l}\text { VIEJAS CLASES } \\
\text { MEDIAS }\end{array}$ & $\begin{array}{l}\text { OBREROS } \\
\text { CUALIFICADOS }\end{array}$ & $\begin{array}{l}\text { OBREROS } \\
\text { NO CUALIFICADOS }\end{array}$ \\
\hline Que se les dé una alimentación correcta y equilibrada & 3,8186 & 3,8247 & 3,7714 & 3,7711 & 3,7838 & 0,128 \\
\hline Que el personal sea cariñoso y afectuoso & 3,7710 & 3,8062 & 3,7775 & 3,7806 & 3,8081 & 0,632 \\
\hline Que los locales sean amplios y en buenas condiciones & 3,7172 & 3,7423 & 3,6792 & 3,6821 & 3,7003 & 0,202 \\
\hline Que el personal tenga un título oficial & 3,6765 & 3,6818 & 3,6212 & 3,6568 & 3,6149 & 0,335 \\
\hline Que haya un programa educativo espećfíco & 3,6159 & 3,6485 & 3,5849 & 3,5496 & 3,5625 & 0,041 \\
\hline Que ofrezca flexibilidad de horarios & 3,5432 & 3,5560 & 3,5325 & 3,5241 & 3,5768 & 0,740 \\
\hline Que haya pocos niños en el aula & 3,5000 & 3,5509 & 3,4330 & 3,4578 & 3,4660 & 0,044 \\
\hline Que tenga servicio de transporte & 3,1793 & 3,1896 & 3,3325 & 3,3007 & 3,2983 & 0,003 \\
\hline Que ofrezca la enseñanza de idiomas & 3,0594 & 3,0549 & 3,0106 & 3,0226 & 3,0375 & 0,913 \\
\hline
\end{tabular}

Nota: La escala se ha re-codificado de manera que 1 es nada importante y 4 muy importante.

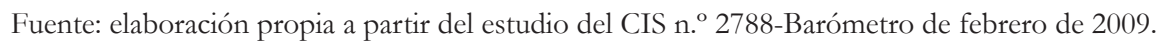

Las tablas 28 a 31 muestran sus preferencias cuando se les pide que elijan la más importante y la segunda en importancia del listado anterior (v21). Si miramos al cruzar estas repuestas con la ideología declarada se aprecia que se mantiene el orden de las anteriores elecciones, y las diferencias por ideología no son significativas. Por su parte, las diferencias entre clases sociales sí son significativas, pero presentan pocas variaciones de orden entre clases sociales sino que se deben en mayor medida a la intensidad, así «que el personal tenga un título oficial» importa como primera opción a todas las clases en primer lugar, pero con mayor intensidad a la Clase alta/media-alta y a las Nuevas clases medias.

Tabla XXVIII. Respuesta a la pregunta: de todas las características que le acabo de mencionar, ¿cuál sería la más importante para Ud. a la hora de elegir un centro para un niño o una niña menor de 3 años? (v21) en función de la ideología (v27)

\begin{tabular}{lll}
\hline & IZQUIERDA & DERECHA \\
\hline Que el personal tenga un título oficial & $34,9 \%$ & $40,2 \%$ \\
\hline Que el personal sea cariñoso y afectuoso & $26,1 \%$ & $21,3 \%$ \\
\hline Que haya un programa educativo específico & $11,0 \%$ & $12,9 \%$ \\
\hline Que los locales sean amplios y en buenas condiciones & $8,8 \%$ & $7,4 \%$ \\
\hline Que se les dé una alimentación correcta y equilibrada & $6,4 \%$ & $7,8 \%$ \\
\hline Que haya pocos niños en el aula & $5,6 \%$ & $4,9 \%$ \\
\hline Que ofrezca flexibilidad de horarios & $4,8 \%$ & $3,4 \%$ \\
\hline Que ofrezca la enseñanza de idiomas & $1,1 \%$ & $1,1 \%$ \\
\hline
\end{tabular}




\begin{tabular}{lll}
\hline & IZQUIERDA & DERECHA \\
\hline Otras características & $0,6 \%$ & $0,6 \%$ \\
\hline Que tenga servicio de transporte & $0,6 \%$ & $0,4 \%$ \\
\hline
\end{tabular}

Pearson Chi-Cuadrado $=12,001 ; \mathrm{gl}=9 ;$ Sig. $=0,213$

Fuente: elaboración propia a partir del estudio del CIS n. ${ }^{\circ}$ 2788-Barómetro de febrero de 2009.

Tabla XXIX. Respuesta a la pregunta: ¿y la segunda en importancia? (v21) en función de la ideología (v27)

\begin{tabular}{lll}
\hline & IZQUIERDA & DERECHA \\
\hline Que el personal tenga un título oficial & $21,9 \%$ & $20,8 \%$ \\
\hline Que el personal sea cariñoso y afectuoso & $19,4 \%$ & $17,0 \%$ \\
\hline Que haya un programa educativo específico & $13,7 \%$ & $15,7 \%$ \\
\hline Que los locales sean amplios y en buenas condiciones & $13,4 \%$ & $12,0 \%$ \\
\hline Que se les dé una alimentación correcta y equilibrada & $12,0 \%$ & $11,7 \%$ \\
\hline Que haya pocos niños en el aula & $8,1 \%$ & $9,0 \%$ \\
\hline Que ofrezca flexibilidad de horarios & $8,0 \%$ & $8,0 \%$ \\
\hline Que ofrezca la enseñanza de idiomas & $1,9 \%$ & $3,4 \%$ \\
\hline Que tenga servicio de transporte & $1,1 \%$ & $1,9 \%$ \\
\hline Otras características & $0,5 \%$ & $0,4 \%$ \\
\hline
\end{tabular}

Pearson Chi-Cuadrado $=9,009 ;$ gl $=9 ;$ Sig. $=0,436$

Fuente: elaboración propia a partir del estudio del CIS n. ${ }^{\circ}$ 2788-Barómetro de febrero de 2009.

Tabla XXX. Respuesta a la pregunta: de todas las características que le acabo de mencionar, ¿cuál sería la más importante para Ud. a la hora de elegir un centro para un niño o una niña menor de 3 años? (v21) en función de la clase social (v44)

\begin{tabular}{llllll} 
& $\begin{array}{l}\text { CLASE ALTA/ } \\
\text { MEDIA-ALTA }\end{array}$ & $\begin{array}{l}\text { NUEVAS CLASES } \\
\text { MEDIAS }\end{array}$ & $\begin{array}{l}\text { VIEJAS CLASES } \\
\text { MEDIAS }\end{array}$ & $\begin{array}{l}\text { OBREROS } \\
\text { CUALIFICADOS }\end{array}$ & $\begin{array}{l}\text { OBREROS } \\
\text { NO CUALIFICADOS }\end{array}$ \\
\hline Que el personal tenga un fítulo oficial & $36,5 \%$ & $36,9 \%$ & $34,3 \%$ & $35,9 \%$ & $31,7 \%$ \\
\hline Que el personal sea cariñoso y afectuoso & $25,6 \%$ & $25,8 \%$ & $25,1 \%$ & $26,1 \%$ & $27,0 \%$ \\
\hline Que haya un programa educativo espećtico & $15,1 \%$ & $11,0 \%$ & $7,9 \%$ & $9,3 \%$ & $10,9 \%$ \\
\hline Que los locales sean amplios y en buenas condiciones & $6,2 \%$ & $8,1 \%$ & $12,4 \%$ & $7,7 \%$ & $10,6 \%$ \\
\hline Que se les dé una alimentación correcta y equilibrada & $3,4 \%$ & $5,8 \%$ & $8,2 \%$ & $9,4 \%$ & $10,6 \%$ \\
\hline Que haya pocos niños en el aula & $6,4 \%$ & $4,6 \%$ & $5,0 \%$ & $5,3 \%$ & $4,1 \%$ \\
\hline Que ofrezca flexibilidad de horarios & $3,9 \%$ & $5,4 \%$ & $4,0 \%$ & $3,8 \%$ & $4,4 \%$ \\
\hline Que ofrezca la enseñanza de idiomas & $1,8 \%$ & $1,0 \%$ & $1,1 \%$ & $1,2 \%$ & $0,7 \%$ \\
\hline Que tenga servicio de transporte & $0,7 \%$ & $0,2 \%$ & $1,1 \%$ & $0,7 \%$ & $0,3 \%$ \\
\hline Que ofrezca la enseñanza de idiomas & $0,5 \%$ & $1,0 \%$ & $1,1 \%$ & $0,5 \%$ & $0,3 \%$ \\
\hline
\end{tabular}

Pearson Chi-Cuadrado $=60,123 ; \mathrm{gl}=36$; Sig. $=0,007$

Fuente: elaboración propia a partir del estudio del CIS n. ${ }^{\circ}$ 2788-Barómetro de febrero de 2009.

Tabla XXXI. Respuesta a la pregunta: ¿y la segunda en importancia? (v21) en función de la clase social (v44)

\begin{tabular}{llllll}
\hline & $\begin{array}{l}\text { CLASE ALTA/ } \\
\text { MEDIA-ALTA }\end{array}$ & $\begin{array}{l}\text { NUEVAS CLASES } \\
\text { MEDIAS }\end{array}$ & $\begin{array}{l}\text { VIEJAS CLASES } \\
\text { MEDIAS }\end{array}$ & $\begin{array}{l}\text { OBREROS } \\
\text { CUALIFICADOS }\end{array}$ & $\begin{array}{l}\text { OBREROS } \\
\text { NO CUALIFICADOS }\end{array}$ \\
\hline Que el personal sea cariñoso y afectuoso & $18,0 \%$ & $20,6 \%$ & $20,9 \%$ & $23,9 \%$ & $22,8 \%$ \\
\hline Que se les dé una alimentación correcta y equilibrada & $16,4 \%$ & $19,8 \%$ & $20,9 \%$ & $21,3 \%$ & $20,4 \%$ \\
\hline Que los locales sean amplios y en buenas condiciones & $14,2 \%$ & $16,2 \%$ & $14,4 \%$ & $15,4 \%$ & $10,0 \%$ \\
\hline Que el personal tenga un título oficial & $13,0 \%$ & $11,6 \%$ & $13,4 \%$ & $11,9 \%$ & $17,3 \%$
\end{tabular}




\begin{tabular}{llllll}
\hline & $\begin{array}{l}\text { CLASE ALTA/ } \\
\text { MEDIA-ALTA }\end{array}$ & $\begin{array}{l}\text { NUEVAS CLASES } \\
\text { MEDIAS }\end{array}$ & $\begin{array}{l}\text { VIEJAS CLASES } \\
\text { MEDIAS }\end{array}$ & $\begin{array}{l}\text { OBREROS } \\
\text { CUALIFICADOS }\end{array}$ & $\begin{array}{l}\text { OBREROS } \\
\text { NO CUALIFICADOS }\end{array}$ \\
\hline Que haya un programa educativo espećfico & $13,5 \%$ & $13,7 \%$ & $11,0 \%$ & $9,8 \%$ & $10,7 \%$ \\
\hline Que ofrezca flexibilidad de horarios & $9,6 \%$ & $6,5 \%$ & $8,8 \%$ & $8,0 \%$ & $6,9 \%$ \\
\hline Que haya pocos niños en el aula & $10,7 \%$ & $8,6 \%$ & $7,0 \%$ & $5,6 \%$ & $6,2 \%$ \\
\hline Que ofrezca la enseñanza de idiomas & $3,2 \%$ & $2,5 \%$ & $1,1 \%$ & $2,0 \%$ & $2,1 \%$ \\
\hline Que tenga servicio de transporte & $0,7 \%$ & $0,2 \%$ & $2,4 \%$ & $1,5 \%$ & $2,1 \%$ \\
\hline Otras características & $0,7 \%$ & $0,2 \%$ & $0,3 \%$ & $0,5 \%$ & $1,4 \%$ \\
\hline
\end{tabular}

Pearson Chi-Cuadrado $=60,123 ; \mathrm{gl}=36$; Sig. $=0,007$

Fuente: elaboración propia a partir del estudio del CIS n. ${ }^{\circ}$ 2788-Barómetro de febrero de 2009.

\section{c) Sobre la provisión de educación infantil}

En las tablas 32 y 33 se puede observar, de nuevo por ideología y clase social, el sentido de la respuesta a la pregunta sobre qué tipo de centro cree que proporciona una mejor educación infantil. La mayor significación la encontramos en su cruce con la variable ideología, dónde en la izquierda predomina la preferencia por la provisión pública del servicio (48\%) mientras que en la derecha predomina la preferencia por un centro privado $(45,4 \%)$. En todos los grupos por clase social la respuesta más frecuente es un centro público, y en segundo lugar un centro privado (la tercera opción «ambos por igual» no era leída, por lo que solo se marcaba a iniciativa de la encuestada o encuestado). No obstante, la preferencia de la pública sobre la privada se incrementa a medida que se desciende de case social analizada desde la clase alta/media alta $(11 \%)$ a obreros no cualificados $(17,5 \%)$, a excepción de las nuevas clases medias que marcan la menor de las diferencias $(0,9 \%)$.

Tabla XXXII. Respuesta a la pregunta: ¿qué tipo de centro cree Ud. que proporciona una mejor educación infantil, un centro público o un centro privado? (v22) en función de la ideología (v27)

Pearson Chi-Cuadrado $=57,279 ; \mathrm{gl}=2$; Sig. $=0,000$

\begin{tabular}{lll}
\hline & IZQUIERDA & DERECHA \\
\hline Centro público & $48,0 \%$ & $32,6 \%$ \\
\hline Centro privado & $26,5 \%$ & $45,4 \%$ \\
\hline Ambos por igual & $25,4 \%$ & $22,0 \%$ \\
\hline
\end{tabular}

Fuente: elaboración propia a partir del estudio del CIS n. ${ }^{\circ}$ 2788-Barómetro de febrero de 2009.

Tabla XXXIII. Respuesta a la pregunta: ¿qué tipo de centro cree Ud. que proporciona una mejor educación infantil, un centro público o un centro privado? (v22) en función de la clase social (v44)

\begin{tabular}{|c|c|c|c|c|c|}
\hline & $\begin{array}{l}\text { CLASE ALTA/ } \\
\text { MEDIA-ALTA }\end{array}$ & $\begin{array}{l}\text { NUEVAS CLASES } \\
\text { MEDIAS }\end{array}$ & $\begin{array}{l}\text { VIEJAS CLASES } \\
\text { MEDIAS }\end{array}$ & $\begin{array}{l}\text { OBREROS } \\
\text { CUALIFICADOS }\end{array}$ & $\begin{array}{l}\text { OBREROS } \\
\text { NO CUALIFICADOS }\end{array}$ \\
\hline Centro público & $44,4 \%$ & $37,9 \%$ & $42,4 \%$ & $43,5 \%$ & $45,9 \%$ \\
\hline Centro privado & $33,3 \%$ & $36,9 \%$ & $29,3 \%$ & $29,2 \%$ & $28,4 \%$ \\
\hline Ambos por igual & $22,3 \%$ & $25,2 \%$ & $28,4 \%$ & $27,3 \%$ & $25,7 \%$ \\
\hline
\end{tabular}

Pearson Chi-Cuadrado = 13,355; gl = 8; Sig. $=0,100$

Fuente: elaboración propia a partir del estudio del CIS n. ${ }^{\circ}$ 2788-Barómetro de febrero de 2009.

\section{d) Problemáticas identificadas en la escolarización 0-2 años}

Por otro lado, tablas 34 a 37, cuando son preguntados acerca de cuál es el principal problema en relación a las escuelas infantiles o guarderías para menores de 3 años (v23), el principal proble- 
ma señalado es la oferta de plazas, ya que indican que no hay centros públicos suficientes para todos los niños el 33,9\%). Un segundo bloque de problemas es que los centros privados son demasiado caros $(13,1 \%)$, que no hay suficientes profesionales cualificados $(12,0 \%)$, que hay demasiados niños por aula $(10,3 \%)$ o que hay centros que no cumplen con los requisitos mínimos $(10,0 \%)$. Por último, otras cuestiones menores es que los centros no ofrecen flexibilidad de horarios $(5,2 \%)$ o que solo consiguen plazas las familias con rentas muy bajas (4,1\%). Aunque no varían mucho el orden de las preferencias, si lo hace su intensidad, siendo las diferencias de las cuatro tablas significativas, están ordenadas por su distancia en intensidad para facilitar su lectura.

Tabla XXXIV. Respuesta a la pregunta: ¿cuál es el principal problema en relación a las escuelas infantiles o guarderías para menores de 3 años? (v23) en función de la ideología (v27)

\begin{tabular}{lll}
\hline & IZQUIERDA & DERECHA \\
\hline No hay centros públicos suficientes para todos los niños & $41,9 \%$ & $33,0 \%$ \\
\hline Hay demasiados niños por aula & $10,1 \%$ & $12,5 \%$ \\
\hline Los centros privados son demasiado caros & $13,0 \%$ & $14,9 \%$ \\
\hline Los centros no ofrecen flexibilidad de horarios & $5,7 \%$ & $7,0 \%$ \\
\hline Hay centros que no cumplen con los requisitos mínimos & $11,1 \%$ & $12,5 \%$ \\
\hline No hay suficientes profesionales cualificados & $13,0 \%$ & $14,1 \%$ \\
\hline Otros problemas & $0,7 \%$ & $1,4 \%$ \\
\hline Solo consiguen plazas las familias con rentas muy bajas & $4,6 \%$ & $4,6 \%$ \\
\hline
\end{tabular}

Pearson Chi-Cuadrado = 14,279; $\mathrm{gl}=2$; Sig. $=0,000$

Fuente: elaboración propia a partir del estudio del CIS n. ${ }^{\circ}$ 2788-Barómetro de febrero de 2009.

Tabla XXXV. Respuesta a la pregunta: ¿Y el segundo? (v23) en función de la ideología (v27)

\begin{tabular}{lll}
\hline & IZQUIERDA & DERECHA \\
\hline Los centros privados son demasiado caros & $28,3 \%$ & $20,6 \%$ \\
\hline Solo consiguen plazas las familias con rentas muy bajas & $9,7 \%$ & $15,0 \%$ \\
\hline No hay centros públicos suficientes para todos los niños & $19,9 \%$ & $23,4 \%$ \\
\hline Los centros no ofrecen flexibilidad de horarios & $8,6 \%$ & $5,6 \%$ \\
\hline No hay suficientes profesionales cuallificados & $8,3 \%$ & $10,3 \%$ \\
\hline Hay demasiados niños por aula & $12,0 \%$ & $10,5 \%$ \\
\hline Hay centros que no cumplen con los requisitos mínimos & $12,1 \%$ & $13,1 \%$ \\
\hline Otros problemas & $1,1 \%$ & $1,5 \%$ \\
\hline
\end{tabular}

Pearson Chi-Cuadrado $=24,703 ; \mathrm{gl}=7$; Sig. $=0,001$

Fuente: elaboración propia a partir del estudio del CIS n. ${ }^{\circ}$ 2788-Barómetro de febrero de 2009.

Tabla XXXVI. Respuesta a la pregunta: ¿cuál es el principal problema en relación a las escuelas infantiles o guarderías para menores de 3 años? (v23) en función de la clase social (v44)

\begin{tabular}{llllll}
\hline & $\begin{array}{l}\text { CLASE ALTA/ } \\
\text { MEDIA-ALTA }\end{array}$ & $\begin{array}{l}\text { NUEVAS CLASES } \\
\text { MEDIAS }\end{array}$ & $\begin{array}{l}\text { VIEJAS CLASES } \\
\text { MEDIAS }\end{array}$ & $\begin{array}{l}\text { OBREROS } \\
\text { CUALIFICADOS }\end{array}$ & $\begin{array}{l}\text { OBREROS } \\
\text { NO CUALIFICADOS }\end{array}$ \\
\hline No hay centros públicos suficientes para todos los niños & $43,2 \%$ & $34,7 \%$ & $35,0 \%$ & $38,5 \%$ & $36,2 \%$ \\
\hline Los centros privados son demasiado caros & $10,7 \%$ & $15,3 \%$ & $17,6 \%$ & $16,3 \%$ & $11,2 \%$ \\
\hline No hay suficientes profesionales cualificados & $12,1 \%$ & $12,0 \%$ & $17,1 \%$ & $12,9 \%$ & $13,4 \%$ \\
\hline Hay centros que no cumplen con los requisitos mínimos & $12,4 \%$ & $13,1 \%$ & $8,7 \%$ & $10,1 \%$ & $13,0 \%$ \\
\hline Hay demasiados niños por aula & $8,1 \%$ & $13,6 \%$ & $11,6 \%$ & $12,0 \%$ & $13,4 \%$ \\
\hline
\end{tabular}




\begin{tabular}{llllll}
\hline & $\begin{array}{l}\text { CLASE ALTA/ } \\
\text { MEDIA-ALTA }\end{array}$ & $\begin{array}{l}\text { NUEVAS CLASES } \\
\text { MEDIAS }\end{array}$ & $\begin{array}{l}\text { VIEJAS CLASES } \\
\text { MEDIAS }\end{array}$ & $\begin{array}{l}\text { OBREROS } \\
\text { CUALIFICADOS }\end{array}$ & $\begin{array}{l}\text { OBREROS } \\
\text { NO CUALIFICADOS }\end{array}$ \\
\hline Los centros no ofrecen flexibilidad de horarios & $8,3 \%$ & $6,2 \%$ & $4,3 \%$ & $5,0 \%$ & $6,2 \%$ \\
\hline Solo consiguen plazas las familias con rentas muy bajas & $5,0 \%$ & $4,4 \%$ & $4,6 \%$ & $4,2 \%$ & $5,8 \%$ \\
\hline Otros problemas & $0,2 \%$ & $0,7 \%$ & $1,2 \%$ & $1,0 \%$ & $0,7 \%$ \\
\hline
\end{tabular}

Pearson Chi-Cuadrado $=43,306 ; \mathrm{gl}=28 ;$ Sig. $=0,033$

Fuente: elaboración propia a partir del estudio del CIS n. ${ }^{\circ}$ 2788-Barómetro de febrero de 2009.

Tabla XXXVII. Respuesta a la pregunta: ¿Y el segundo? (v23) en función de la clase social (v44)

\begin{tabular}{llllll}
\hline & $\begin{array}{l}\text { CLASE ALTA/ } \\
\text { MEDIA-ALTA }\end{array}$ & $\begin{array}{l}\text { NUEVAS CLASES } \\
\text { MEDIAS }\end{array}$ & $\begin{array}{l}\text { VIEJAS CLASES } \\
\text { MEDIAS }\end{array}$ & $\begin{array}{l}\text { OBREROS } \\
\text { CUALIFICADOS }\end{array}$ & $\begin{array}{l}\text { OBREROS } \\
\text { NO CUALIFICADOS }\end{array}$ \\
\hline Los centros privados son demasiado caros & $28,4 \%$ & $23,3 \%$ & $26,2 \%$ & $26,6 \%$ & $33,7 \%$ \\
\hline No hay centros públicos suficientes para todos los niños & $16,8 \%$ & $23,3 \%$ & $22,0 \%$ & $23,0 \%$ & $21,5 \%$ \\
\hline No hay suticientes profesionales cualificados & $13,3 \%$ & $12,0 \%$ & $16,0 \%$ & $10,2 \%$ & $9,2 \%$ \\
\hline Hay demasiados niños por aula & $12,8 \%$ & $11,1 \%$ & $8,9 \%$ & $13,2 \%$ & $7,7 \%$ \\
\hline Hay centros que no cumplen con los requisitos mínimos & $10,4 \%$ & $14,1 \%$ & $8,3 \%$ & $9,9 \%$ & $11,5 \%$ \\
\hline Solo consiguen plazas las familias con rentas muy bajas & $9,6 \%$ & $8,3 \%$ & $9,6 \%$ & $8,2 \%$ & $7,3 \%$ \\
\hline Los centros no ofrecen flexibilidad de horarios & $7,4 \%$ & $7,6 \%$ & $7,7 \%$ & $7,3 \%$ & $7,3 \%$ \\
\hline Otros problemas & $1,2 \%$ & $0,2 \%$ & $1,3 \%$ & $1,6 \%$ & $1,9 \%$ \\
\hline
\end{tabular}

Pearson Chi-Cuadrado $=41,490 ; \mathrm{gl}=28$; Sig. $=0,048$

Fuente: elaboración propia a partir del estudio del CIS n. ${ }^{2}$ 2788-Barómetro de febrero de 2009.

Por su parte las tablas 38 y 39 muestran las que, en opinión de las y los encuestados, deberían ser las prioridades de la Administración en relación con la educación infantil. De nuevo aquí nos encontramos con que las diferencias más que de orden lo son de intensidad, y de manera significativa. Los encuestados de izquierdas se decantan en mayor medida por la creación de más escuelas infantiles y menos que los encuestados de derechas por promover convenios y subvenciones. De igual modo, si bien todas las clases sociales se decantan por crear más escuelas, las segundas y terceras preferencias varían. Así la clase alta/media-alta tiende a preferir como segunda y tercera opción por promover convenios y subvenciones, las otras suelen decantarse antes por las ayudas económicas directas a las familias.

Tabla XXXVIII. Respuesta a la pregunta: De las siguientes medidas que ponen en marcha las Administraciones Públicas, ¿cuál le parece prioritaria para proporcionar atención educativa a los niños y las niñas menores de 3 años? (v24) en función de la ideología (v27)

\begin{tabular}{lll}
\hline & IZQUIERDA & DERECHA \\
\hline Crear más escuelas infantiles & $45,6 \%$ & $36,8 \%$ \\
\hline Ayudas económicas directas a las familias & $24,8 \%$ & $23,7 \%$ \\
\hline Promover convenios para poner escuelas en centros de trabajo & $16,9 \%$ & $19,7 \%$ \\
\hline Subvencionar más escuelas infantiles & $12,7 \%$ & $19,8 \%$ \\
\hline
\end{tabular}

Pearson Chi-Cuadrado $=21,304 ; \mathrm{gl}=3$; Sig. $=0,000$

Fuente: elaboración propia a partir del estudio del CIS n. ${ }^{\circ}$ 2788-Barómetro de febrero de 2009. 
Tabla XXXIX. Respuesta a la pregunta: De las siguientes medidas que ponen en marcha las Administraciones Públicas, ¿cuál le parece prioritaria para proporcionar atención educativa a los niños y las niñas menores de 3 años? (v24) en función de la clase social (v44)

\begin{tabular}{llllll}
\hline & CLASE ALTA/ & NUEVAS CLASES & VIEJAS CLASES & OBREROS & OBREROS \\
& MEDIA-ALTA & MEDIAS & MEDIAS & CUALIFICADOS & NO CUALIFICADOS \\
\hline Crear más escuelas infantiles & $43,4 \%$ & $42,9 \%$ & $41,2 \%$ & $41,9 \%$ & $44,1 \%$ \\
\hline Ayudas económicas directas a las familias & $15,3 \%$ & $20,7 \%$ & $32,8 \%$ & $29,9 \%$ & $29,5 \%$ \\
\hline Promover convenios para poner escuelas en centros de trabajo & $24,1 \%$ & $20,7 \%$ & $10,5 \%$ & $15,3 \%$ & $15,6 \%$ \\
\hline Subvencionar más escuelas infantiles & $17,2 \%$ & $15,6 \%$ & $15,5 \%$ & $13,0 \%$ & $10,8 \%$ \\
\hline
\end{tabular}

Pearson Chi-Cuadrado $=71,297 ; \mathrm{gl}=12 ;$ Sig. $=0,000$

Fuente: elaboración propia a partir del estudio del CIS n. ${ }^{\circ}$ 2788-Barómetro de febrero de 2009.

\section{e) Sobre las prócticas de crianza}

A la pregunta «iTiene $U d$. hijos/as?» (v25) contestaron afirmativamente 1710 personas, lo que equivale al 68,7\% de la muestra original. Es a esta sub-muestra, la de madres y padres, a quienes se les preguntó acerca si «su bijo/a menor, zantes de los 3 años asistió (asiste) su hijo/ a a algún centro de atención a la infancia (guardería, jardín de infancia) o escuela infantila) (v26) y, en función de si asistió (asiste) o no, las razones de por qué lo hizo (v26a y v26b). Veamos a continuación la tabla 40, en ella se aprecia que es mayoritario el número de madres y padres encuestados que no llevaron (llevan) a su hija o hijo a un centro de educación infantil de primer ciclo, el 57,5\% frente al $41,2 \%$ que si lo hicieron (hacen).

Tabla XXXX. Distribución de la respuesta a la pregunta: ¿antes de los 3 años asistió (asiste) su hijo/a a algún centro de atención a la infancia (guardería, jardín de infancia) o escuela infantil? (v26)

\begin{tabular}{lll}
\hline & $\mathrm{N}^{\circ}$ & $\%$ \\
\hline $\mathrm{Si}$ & 705 & 41,2 \\
\hline No & 983 & 57,5 \\
\hline N.C. & 22 & 1,3 \\
\hline Total & 1710 & 100,0 \\
\hline
\end{tabular}

Fuente: elaboración propia a partir del estudio del CIS n. ${ }^{\circ}$ 2788-Barómetro de febrero de 2009.

Veamos a continuación si las diferencias entre grupos, en función de la ideología y la clase social. Los resultados (tabla 41) muestran, de manera significativa, las diferencias entre personas de ideologías de derecha, menos proclives a matricular a los menores de 3 años en centros de educación infantil (61,5\% frente a 54,9\%). Estas diferencias (tabla 42) son mucho más marcadas entre la clase alta/ media-alta y las nuevas clases medias (42,9\% y 50,5\% respectivamente) que las viejas clases medias, los obreros cualificados y los obreros no cualificados más tendentes a no matricularlos $(65,3 \%, 63,7 \%$ y $61,5 \%$ respectivamente). 
Tabla XXXXI. Distribución de la respuesta a la pregunta: ¿antes de los 3 años asistió (asiste) su hijo/a a algún centro de atención a la infancia (guardería, jardín de infancia)

o escuela infantil? (v26) en función de la ideología (v27)

\begin{tabular}{lll}
\hline & IZQUIERDA & DERECHA \\
\hline $\mathrm{Si}$ & $45,1 \%$ & $38,5 \%$ \\
\hline No & $54,9 \%$ & $61,5 \%$ \\
\hline
\end{tabular}

Pearson Chi-Cuadrado = 4,852; $\mathrm{gl}=1$; Sig. $=0,028$

Fuente: elaboración propia a partir del estudio del CIS n. ${ }^{\circ}$ 2788-Barómetro de febrero de 2009.

Tabla XXXXII. Distribución de la respuesta a la pregunta: ¿antes de los 3 años asistió (asiste) su hijo/a a algún centro de atención a la infancia (guardería, jardín de infancia) o escuela infantil? (v26) en función de la clase social (v44)

\begin{tabular}{llllll}
\hline & CLASE ALTA/ & NUEVAS CLASES & VIEJAS CLASES & OBREROS & OBREROS \\
& MEDIA-ALTA & MEDIAS & MEDIAS & CUALIFICADOS & NO CUALIFICADOS \\
\hline $\mathrm{Si}$ & $57,1 \%$ & $49,5 \%$ & $34,7 \%$ & $36,3 \%$ & $38,5 \%$ \\
\hline $\mathrm{No}$ & $42,9 \%$ & $50,5 \%$ & $65,3 \%$ & $63,7 \%$ & $10,8 \%$ \\
\hline
\end{tabular}

Pearson Chi-Cuadrado $=45,611 ; \mathrm{gl}=4$; Sig. $=0,000$

Fuente: elaboración propia a partir del estudio del CIS n. ${ }^{\circ}$ 2788-Barómetro de febrero de 2009.

\section{f) Acerca de la justificación de la escolarización o no escolarización}

Para acabar esta sección del análisis, veamos por último la justificación alegada a la asistencia o no asistencia de su hijo o hija menor a un centro de educación infantil de primer ciclo. En la primera pregunta «ipor qué razón asistió (asiste) su hijo/a a una escuela infantil o guardería?» (v26a) las razones alegadas son coincidentes a ambos lados del espectro ideológico. Las principales son «Porque yo (y mi cónyuge) trabajamos», «Para que conviva con los demás» y «Para que aprenda y se desarrolle». Ni las diferencias entre ideología (tabla 43) o entre clases sociales (tabla 44) son significativas.

Tabla XXXXIII. Respuesta a la pregunta: ¿por qué razón asistió (asiste) su hijo/a a una escuela infantil o guardería? (v26a) en función de la ideología (v27)

\begin{tabular}{lll}
\hline & IZQUIERDA & DERECHA \\
\hline Porque yo (y mi cónyuge) trabajamos & $61,8 \%$ & $51,7 \%$ \\
\hline Para que conviva con los demás & $17,6 \%$ & $23,8 \%$ \\
\hline Para que aprenda y se desarrolle & $15,0 \%$ & $17,9 \%$ \\
\hline Otra razón & $2,5 \%$ & $4,0 \%$ \\
\hline Porque no tengo familiares que le pudieran cuidar & $3,1 \%$ & $2,6 \%$ \\
\hline
\end{tabular}

Pearson Chi-Cuadrado = 5,532; gl = 4; Sig. $=0,237$

Fuente: elaboración propia a partir del estudio del CIS n. ${ }^{\circ}$ 2788-Barómetro de febrero de 2009.

Tabla XXXXIV. Respuesta a la pregunta: ¿por qué razón asistió (asiste) su hijo/a a una escuela infantil o guardería? (v26a) en función de la clase social (v44)

\begin{tabular}{llllll}
\hline & $\begin{array}{l}\text { CLASE ALTA/ } \\
\text { MEDIA-ALTA }\end{array}$ & $\begin{array}{l}\text { NUEVAS CLASES } \\
\text { MEDIAS }\end{array}$ & $\begin{array}{l}\text { VIEJAS CLASES } \\
\text { MEDIAS }\end{array}$ & $\begin{array}{l}\text { OBREROS } \\
\text { CUALIFICADOS }\end{array}$ & $\begin{array}{l}\text { OBREROS } \\
\text { NO CUALIFICADOS }\end{array}$ \\
\hline Porque yo (y mi cónyuge) trabajamos & $58,6 \%$ & $59,7 \%$ & $56,6 \%$ & $54,9 \%$ & $71,4 \%$ \\
\hline Para que conviva con los demás & $20,0 \%$ & $16,9 \%$ & $21,2 \%$ & $21,6 \%$ & $14,3 \%$ \\
\hline Para que aprenda y se desarrolle & $12,9 \%$ & $16,9 \%$ & $17,2 \%$ & $16,7 \%$ & $9,5 \%$ \\
\hline
\end{tabular}




\begin{tabular}{llllll}
\hline & $\begin{array}{l}\text { CLASE ALTA/ } \\
\text { MEDIA-ALTA }\end{array}$ & $\begin{array}{l}\text { NUEVAS CLASES } \\
\text { MEDIAS }\end{array}$ & $\begin{array}{l}\text { VIEJAS CLASES } \\
\text { MEDIAS }\end{array}$ & $\begin{array}{l}\text { OBREROS } \\
\text { CUALIFICADOS }\end{array}$ & $\begin{array}{l}\text { OBREROS } \\
\text { NO CUALIFICADOS }\end{array}$ \\
\hline Otra razón & $3,6 \%$ & $3,9 \%$ & $3,0 \%$ & $4,9 \%$ & $3,6 \%$ \\
\hline Porque no tengo familiares que le pudieran cuidar & $5,0 \%$ & $2,6 \%$ & $2,0 \%$ & $2,0 \%$ & $1,2 \%$ \\
\hline
\end{tabular}

Pearson Chi-Cuadrado = 13,236; $\mathrm{gl}=16$; Sig. $=0,655$

Fuente: elaboración propia a partir del estudio del CIS n. ${ }^{\circ}$ 2788-Barómetro de febrero de 2009.

Tampoco son significativas las diferencias por ideología (Tabla 45) respecto de la pregunta «¿por qué razón no asistió (asiste) su hijo/a a una escuela infantil o guardería?» (v26b) donde la prelación es "Porque yo (y mi cónyuge) no trabajamos", "Porque (antes) no existían centros de ningún tipo" y "Porque prefiero que le cuiden mis familiares". No obstante respecto de ésta pregunta las diferencias entre clases (Tabla 46) sí son significativas con una preeminencia de los Obreros cualificados por la primera de las razones, destacando las Viejas clases medias por la segunda y la Clase alta/media-alta por la tercera.

\section{Tabla XXXXIV. Respuesta a la pregunta: ¿por qué razón no asistió (asiste) su hijo/a a una escuela infantil o guardería? (v26b) en función de la ideología (v27)}

\begin{tabular}{lll}
\hline & IZQUIERDA & DERECHA \\
\hline Porque yo (y mi cónyuge) no trabajamos & $63,2 \%$ & $67,5 \%$ \\
\hline Porque (antes) no existían centros de ningún tipo & $15,1 \%$ & $13,6 \%$ \\
\hline Porque prefiero que le cuiden mis familiares & $11,3 \%$ & $9,5 \%$ \\
\hline Otra razón & $5,0 \%$ & $2,9 \%$ \\
\hline Porque no puedo pagar lo que costaban los centros & $2,7 \%$ & $3,3 \%$ \\
\hline Porque prefiero que le cuide una persona contratada & $2,1 \%$ & $1,6 \%$ \\
\hline Porque no me dieron plaza en ningún centro & $0,6 \%$ & $1,6 \%$ \\
\hline
\end{tabular}

Pearson Chi-Cuadrado = 4,996; $\mathrm{gl}=6$; Sig. $=0,544$

Fuente: elaboración propia a partir del estudio del CIS n. ${ }^{\circ}$ 2788-Barómetro de febrero de 2009.

Tabla XXXXVI. Respuesta a la pregunta: ¿por qué razón no asistió (asiste) su hijo/a a una escuela infantil o guardería? (v26b) en función de la clase social (v44)

\begin{tabular}{llllll}
\hline & $\begin{array}{l}\text { CLASE ALTA/ } \\
\text { MEDIA-ALTA }\end{array}$ & $\begin{array}{l}\text { NUEVAS CLASES } \\
\text { MEDIAS }\end{array}$ & $\begin{array}{l}\text { VIEJAS CLASES } \\
\text { MEDIAS }\end{array}$ & $\begin{array}{l}\text { OBREROS } \\
\text { CUALIFICADOS }\end{array}$ & $\begin{array}{l}\text { OBREROS } \\
\text { NO CUALIFICADOS }\end{array}$ \\
\hline Porque yo (y mi cónyuge) no trabajamos & $60,2 \%$ & $69,0 \%$ & $56,6 \%$ & $71,3 \%$ & $54,9 \%$ \\
\hline Porque (antes) no existían centros de ningún tipo & $5,8 \%$ & $8,9 \%$ & $30,7 \%$ & $11,7 \%$ & $13,5 \%$ \\
\hline Porque prefiero que le cuiden mis familiares & $14,6 \%$ & $7,0 \%$ & $7,4 \%$ & $9,5 \%$ & $15,0 \%$ \\
\hline Otra razón & $9,7 \%$ & $6,3 \%$ & $1,6 \%$ & $3,3 \%$ & $5,3 \%$ \\
\hline Porque no puedo pagar lo que costaban los centros & & $3,8 \%$ & $2,1 \%$ & $2,2 \%$ & $7,5 \%$ \\
\hline Porque prefiero que le cuide una persona contratada & $7,8 \%$ & $2,5 \%$ & $0,5 \%$ & & $2,3 \%$ \\
\hline Porque no me dieron plaza en ningún centro & $1,9 \%$ & $2,5 \%$ & $1,1 \%$ & $1,9 \%$ & $1,5 \%$ \\
\hline Perio
\end{tabular}

Pearson Chi-Cuadrado $=118,102 ; \mathrm{gl}=24 ;$ Sig. $=0,000$

Fuente: elaboración propia a partir del estudio del CIS n. ${ }^{\circ}$ 2788-Barómetro de febrero de 2009.

\section{g) Análisis de regresión}

A continuación, tablas 47 y 48, presentamos los dos análisis de regresión realizados para conocer la fuerza de las variables independientes de nuestro modelo, ideología (v27) y clase social (v44), para explicar la probabilidad matricular (o haber matriculado) a las y los menores de 3 años de edad en un centro de educación infantil de primer ciclo. 
En la tabla 47 se observa que la probabilidad de matricular (o haber matriculado) a las y los menores de 3 años de edad en un centro de educación infantil de primer ciclo se reduce a medida que nos movemos de la izquierda hacia la derecha en el espectro ideológico. Del mismo modo, conforme se incrementa el nivel educativo se incrementan también las probabilidades de matricularla/o. Son resultados robustos, al introducir las variables de control la relación original sigue siendo muy significativa.

Tabla XXXXVII. Análisis de regresión en base a la ideología (v27)

\begin{tabular}{|c|c|c|c|c|c|c|}
\hline & \multicolumn{3}{|c|}{ MODELO 1} & \multicolumn{3}{|c|}{ MODELO 2} \\
\hline & B & $\operatorname{EXP}(B)$ & SIG. & B & $\operatorname{EXP}(B)$ & SIG. \\
\hline izq_der & $-0,074$ & 0,929 & 0,025 & $-0,084$ & 0,920 & 0,022 \\
\hline Mujer & & & & $-0,295$ & 0,745 & 0,033 \\
\hline Edad & & & & $-0,030$ & 0,970 & 0,000 \\
\hline otros miembros hogar & & & & 0,024 & 1,025 & 0,670 \\
\hline español (ref - no nacido en Esp.) & & & & 0,030 & 1,031 & 0,938 \\
\hline \multicolumn{7}{|l|}{ Estudios (Ref = Superior) } \\
\hline Sin estudios & & & & $-0,806$ & 0,447 & 0,063 \\
\hline Primaria & & & & $-0,699$ & 0,497 & 0,029 \\
\hline Secundaria & & & & $-0,532$ & 0,588 & 0,120 \\
\hline F.P. & & & & $-0,253$ & 0,777 & 0,428 \\
\hline Medios universitarios & & & & $-0,253$ & 0,777 & 0,428 \\
\hline \multicolumn{7}{|l|}{ Estatus (Ref = trabajando) } \\
\hline desempleado & & & & $-0,100$ & 0,904 & 0,582 \\
\hline Inactivo & & & & $-0,100$ & 0,904 & 0,582 \\
\hline \multicolumn{7}{|c|}{ Ocupación (Ref = Dirección de empresas y Administraciones Públicas) } \\
\hline Técnicos y profesionales científicos e intelectuales & & & & $-0,038$ & 0,963 & 0,911 \\
\hline Técnicos y profesionales de apoyo & & & & 0,407 & 1,503 & 0,153 \\
\hline Empleados de tipo administrativo & & & & 0,650 & 1,915 & 0,101 \\
\hline Trabajadores de servicios de restauración y personales & & & & $-0,583$ & 0,558 & 0,050 \\
\hline Trabajadores cualificados de agricultura y pesca & & & & $-0,752$ & 0,471 & 0,047 \\
\hline Artesanos y trabajadores cualificados de la industria & & & & $-0,521$ & 0,594 & 0,061 \\
\hline Operadores de maquinaria y montadores & & & & $-0,507$ & 0,602 & 0,076 \\
\hline Trabajadores no cualificados & & & & $-0,539$ & 0,583 & 0,070 \\
\hline Constante & 0,110 & 1,116 & 0,516 & 2,696 & 14,825 & 0,000 \\
\hline Nagelkerke R2 & 0,006 & & & 0,212 & & \\
\hline
\end{tabular}

Fuente: elaboración propia a partir del estudio del CIS n. ${ }^{\circ}$ 2788-Barómetro de febrero de 2009.

Por su parte, en la tabla 48 se observa que a medida que descendemos de clase social lo hacen también las probabilidades de matricular (o haber matriculado) a las y los menores de 3 años de edad en un centro de educación infantil de primer ciclo. La significación de esta relación, como era de esperar, se diluye al introducir en el modelo variables de nivel educativo y ocupación debido a la colinealidad. Es por ello que el poder explicativo de la variable clase social, al introducir las variables de control, desaparece. 
Tabla XXXXVIII. Análisis de regresión en base a clase social (v44)

\begin{tabular}{|c|c|c|c|c|c|c|}
\hline & \multicolumn{3}{|c|}{ MODELO 1} & \multicolumn{3}{|c|}{ MODELO 2} \\
\hline & B & $\operatorname{EXP}(B)$ & SIG. & B & $\operatorname{EXP}(B)$ & SIG. \\
\hline \multicolumn{7}{|l|}{ Estatus (ref - Clase alta) } \\
\hline Nuevas clases medias & $-0,331$ & 0,718 & 0,064 & $-0,172$ & 0,842 & 0,579 \\
\hline Viejas clases medias & $-0,940$ & 0,391 & 0,000 & 0,335 & 1,398 & 0,346 \\
\hline Obreros cualificados & $-0,897$ & 0,408 & 0,000 & 0,208 & 1,232 & 0,567 \\
\hline Obreros no cualificados & $-0,888$ & 0,411 & 0,000 & 0,088 & 1,092 & 0,875 \\
\hline Mujer & & & & $-0,136$ & 0,873 & 0,264 \\
\hline Edad & & & & $-0,031$ & 0,970 & 0,000 \\
\hline otros miembros hogar & & & & 0,026 & 1,026 & 0,595 \\
\hline español (ref - no nacido en Esp.) & & & & 0,389 & 1,476 & 0,249 \\
\hline \multicolumn{7}{|l|}{ Estudios (Ref = Superior) } \\
\hline Sin estudios & & & & $-0,761$ & 0,467 & 0,049 \\
\hline Primaria & & & & $-0,789$ & 0,454 & 0,010 \\
\hline Secundaria & & & & $-0,679$ & 0,507 & 0,037 \\
\hline F.P. & & & & $-0,493$ & 0,610 & 0,121 \\
\hline Medios universitarios & & & & $-0,325$ & 0,722 & 0,280 \\
\hline \multicolumn{7}{|l|}{ Estatus (Ref = trabajando) } \\
\hline desempleado & & & & $-0,136$ & 0,873 & 0,399 \\
\hline Inactivo & & & & $-0,453$ & 0,636 & 0,005 \\
\hline \multicolumn{7}{|c|}{ Ocupación (Ref = Direccion de empresas y Administraciones Publicas) } \\
\hline Técnicos y profesionales cientificos e intelectuales & & & & 0,073 & 1,076 & 0,855 \\
\hline Técnicos y profesionales de apoyo & & & & 0,664 & 1,943 & 0,067 \\
\hline Empleados de tipo administrativo & & & & 0,775 & 2,171 & 0,088 \\
\hline Trabajadores de servicios de restauracion y personales & & & & $-0,285$ & 0,752 & 0,408 \\
\hline Trabajadores cualificados de agricultura y pesca & & & & $-0,680$ & 0,506 & 0,034 \\
\hline Artesanos y trabajadores cualificados de la industria & & & & $-0,393$ & 0,675 & 0,205 \\
\hline Operadores de maquinaria y montadores & & & & $-0,316$ & 0,729 & 0,327 \\
\hline Trabajadores no cualificados & & & & $-0,301$ & 0,740 & 0,607 \\
\hline Constante & 0,380 & 1,462 & 0,005 & 1,755 & 5,782 & 0,002 \\
\hline Nagelkerke R2 & 0,041 & & & 0,187 & & \\
\hline
\end{tabular}

Fuente: elaboración propia a partir del estudio del CIS n. ${ }^{\circ}$ 2788-Barómetro de febrero de 2009.

\section{Conclusiones}

Como se indicaba en la introducción, nos interesaba medir la capacidad de las variables ideología (v27) y clase social (v44) para explicar tendencias de respuesta a las preguntas respecto de la importancia de la escolarización infantil. El análisis de las opiniones respecto la importancia que se otorga a la educación de los niños y niñas menores de 3 años tanto por la sociedad en general (v12) como por las Administraciones Públicas (v13) nos muestra que se percibe un déficit, más acusado para el caso de las Administraciones Públicas en el que un 72,3\% considera que estas le dan «Poca» $\mathrm{O}$ «Ninguna» atención, mientras que cuando hablan de la sociedad en general perciben que esta falta de atención se reduce al 59,2\%. También que la gente que se define como de izquierdas y quienes pertenecen a las «Viejas clases medias», tienden a realizar una valoración algo más positiva de la importancia que otorgan a la educación de los niños y niñas menores de 3 años tanto la sociedad en general como las Administraciones Públicas.

Un resultado hasta cierto punto sorprendente es que se evidencia una extendida confusión entre edad 
de gratuidad (3 años de edad) y edad de escolarización obligatoria (6 años de edad), esta confusión es algo más pronunciada entre las «Viejas clases medias», los «Obreros cualificados», y los «Obreros no cualificados», si bien las diferencias por ideología no son significativas a este respecto. Quizá el hasta cierto punto vaivén normativo en cuanto a la definición de las etapas junto a las elevadas cotas de participación que se dan en tres años y la preeminencia del sector público a partir de tres años, son todos factores que pueden estar alimentando esta confusión.

También se ha observado una tendencia consistente, e independiente del grupo de edad de los niños y niñas, a justificar la conveniencia de la escolarización temprana por razones de conciliación. Este efecto es ligeramente mayor en las clases sociales menos favorecidas, así como en quienes se identifican con posiciones más conservadoras o de derechas. Por el contrario, se ha encontrado mayor tendencia a justificar esa misma conveniencia de escolarización, pero por razones de reducción de las diferencias culturales y sociales, por aquellas clases sociales más favorecidas, y a aquellos quienes se consideran más progresistas o de izquierdas. De nuevo encontramos aquí cierto grado de congruencia con los que los dos principales partidos a ambos lados del espectro ideológico han defendido desde sus legislaciones.

Un resultado algo más previsible indica que las diferencias por adscripción ideológica son evidentes también a la hora del tipo de titularidad del centro ya que para quienes se adscriben a la mitad izquierda del espectro político cree que un centro público proporciona una mejor educación infantil, superando en $15,4 \%$ a quienes eligen esta opción desde la mitad derecha. En efecto, éstos últimos prefieren un centro privado como opción más elegida, un 18,9\% que quienes eligen esta opción desde la mitad izquierda.

Por su parte en el análisis de regresión en base a la ideología se observa que la probabilidad de matricular (o haber matriculado) a las y los menores de 3 años de edad en un centro de educación infantil de primer ciclo se reduce a medida que nos movemos de la izquierda hacia la derecha en el espectro ideológico. Del mismo modo, conforme se incrementa el nivel educativo se incrementan también las probabilidades de matricularla/o. Son éstos resultados robustos y significativos que se mantienen al introducir las variables de control.

Por su parte, en el análisis de regresión en base a clase social se observa que a medida que descendemos de clase social lo hacen también las probabilidades de matricular (o haber matriculado) a las y los menores de 3 años de edad en un centro de educación infantil de primer ciclo. La significación de esta relación, no obstante, se diluye al introducir en el modelo variables de nivel educativo y ocupación debido a la colinealidad. Es por ello que el poder explicativo de la variable clase social, al introducir las variables de control, desaparece.

Así pues, y pesar de plantearse como investigación básica, y hasta cierto punto exploratoria y descriptiva, es también objetivo de este trabajo dar difusión a los resultados de la explotación exhaustiva de la encuesta en la confianza de que no solo contribuirá a un mayor conocimiento acerca del valor que la sociedad las familias otorgan a la educación infantil, sino que también beneficiará a la comunidad educativa en su conjunto. Las Administraciones Públicas encuentran útil conocer mejor cuales son los planteamientos, las necesidades y las preferencias de la sociedad en general, y especialmente de las familias, al respecto de la educación temprana de sus menores, dado que previsiblemente esto les permita adecuar mejor sus políticas. Por su parte, las familias y la comunidad educativa se benefician porque ésta mejor información puede propiciar una oferta educativa más adecuada a sus necesidades y planteamientos. 


\section{Referencias bibliográficas}

Ancheta Arrabal, A. (2011). La escuela infantil hoy. Perspectivas internacionales de la educación y la atención a la primera infancia. Valencia: Tirant.

Bowlby, J. (2005). A secure base. Cronwall: Routledge.

Cebolla-Boado, H.; Radl, J. y Salazar, L. (2014). Aprendiraje y ciclo vital. La desigualdad de oportunidades desde la educación preescolar hasta la edad adulta. Barcelona: Fundación La Caixa.

Colom Ortiz, F. y Gabaldón Estevan, D. (2016): “Recursos educativos y primera infancia: Privatización de la educación infantil de primer ciclo en la ciudad de Valencia". RASE: Revista de la Asociación de Sociología de la Educación, 9 (2), 277-298.

Feito, R. (1994): “¿Debe subvencionarse con fondos públicos la enseñanza privada? Elementos para un debate”. Revista Complutense de Educación, 5 (1), 343.

Fernández-Enguita, M. (2008): “Escuela pública y privada en España: la segregación rampante”. RASE: Revista de la Asociación de Sociología de la Educación, 1 (2).

Fernández-Esquinas, F. (2004): "Elección de escuela: efectos sociales y dilemas en el sistema educativo público en Andalucía". Revista de Educación, 334, 377-390.

Fernández-Palomares, F. (2003): “Socialización y escuela” En Fernández Palomares, F. (coord.) Sociología de la educación. Pearson, Madrid. 206-260.

Gabaldón-Estevan, Daniel (dir.) (2016). Educación y atención a la primera infancia en la ciudad de Valencia. Perfiles familiares ante la elección de centro de primer ciclo de educación infantil. Valencia: Universidad Politécnica de Valencia.

Grana, I. y Martín Zúñiga, F. (2010): “Historia y perspectiva actual de la Educación Infantil” en Carmen Sanchidrián y Julio Ruiz Berrio (Coords.): Políticas educativas de educación infantil en la España Actual (19892009). Barcelona: Graó.

González, M. J. (2005): "Igualando por la base: oportunidades de educación y cuidado de la primera infancia en España" en Vicente Navarro (Ed.): La situación social en España. Madrid: Biblioteca Nueva - Fundación Largo Caballero.

Hendry, J. (1986): "Kindergartens and the Transition from Home to School Education". Comparative Education, $22(1), 53-58$.

Jiménez-Delgado, M.; Jareño-Ruiz, D.; y El-Habib Draoui, B. (2016): “La expansión de la educación infantil en España: entre la igualdad de oportunidades y la segregación”. Revista Iberoamericana De Educación, 72, 19-44. https://doi.org/10.35362/rie72034

Mancebón, M. J. y Pérez-Ximénez, D. (2007): “Conciertos educativos y selección académica y social del alumnado”. Hacienda Pública Española. Revista de Economía Pública, 180 (1), 125-154.

Obiol, S. (2011): "El cambio familiar y el proceso educativo” en José Beltrán, Francesc Jesús Hernàndez. Sociología de la educación. Madrid: McGraw-Hill.

Putnam, R. D. (2016). Our kids: The American Dream in Crisis. New York: Simon and Schuster. 
Rodríguez Pascual, I. (2007). Para una sociología de la infancia, aspectos teóricos y metodológicos. Madrid: CIS

Vila, I. (1995): "Familia y escuela: dos contextos y un solo niño". Aula de innovación educativa, 4 (45), 72-76.

\section{Notas biográficas}

Daniel Gabaldón Estevan es Profesor Titular de Universidad en la Universidad de Valencia, donde imparte sociología de la educación en diferentes grados y máster, cuenta con una amplia experiencia investigadora, y una de sus especializaciones es en temas de educación infantil, relación familias-escuelas y la organización del tiempo de las y los escolares.

Kadri Täht es associate professor en el Instituto de Estudios Sociales Internacionales de la Universidad de Tallin (Estonia). Obtuvo su licenciatura en Sociología de la Universi-dad de Tartu (Estonia); su MA en la Lancaster University (Reino Unido) y Central Eu-ropean University (Polonia); y su Ph.D. en Vrije University Amsterdam (Países Bajos). Kadri Täht se ha especializado en metodologías de investigación, cuantitativas y cualita-tivas, bajo un enfoque eminentemente comparativo a través de su participación en proyectos de investigación nacionales e internacionales y su alto grado de especialización en técnicas de investigación 